\title{
CONTINUOUS COHOMOLOGY AND REAL HOMOTOPY TYPE
}

\author{
EDGAR H. BROWN, JR. AND ROBERT H. SZCZARBA
}

\begin{abstract}
Various aspects of homotopy theory in the category of simplicial spaces are developed. Topics covered include continuous cohomology, continuous de Rham cohomology, the Kan extension condition, the homotopy relation, fibrations, the Serre spectral sequence, real homotopy type and its relation to graded commutative differential algebras over the reals.
\end{abstract}

\section{INTRODUCTION}

In this paper we investigate various aspects of homotopy theory in the category of simplicial spaces, the topics covered being continuous cohomology, continuous de Rham cohomology, the Kan extension condition, the homotopy relation, fibrations, the Serre spectral sequence, real homotopy type and its relation to graded commutative differential algebras over the reals. The paper is organized around stating and proving results which, roughly speaking, establish an equivalence between the category of simplicial spaces localized at the reals and the homotopy category of differential graded commutative topological algebras over the reals. The choice of categories and the formulation of definitions in these categories has been guided by our aim of applying homotopy theory to the spaces $B \Gamma_{q}$ arising in the study of foliations [8] and to secondary characteristic classes of foliations.

In the next section, we state our results and, at the end of the section, give various examples of simplicial spaces occurring in nature.

We are pleased to acknowledge several very helpful conversations with William Dwyer and Dan Kan during the early stages of this work. We are also indebted to Stephen Semmes for assistance with questions relating to linear topological vector spaces.

\section{Statement of Results}

In this section we introduce our main definitions and notation and state our principal results.

For a category $\mathscr{C}, \Delta \mathscr{C}$ will denote the category of simplicial $\mathscr{C}$ 's; $\mathscr{S}$ denotes the category of sets and hence $\Delta \mathscr{S}$ is the category of simplicial sets; $\mathscr{T}$

Received by the editors October 1, 1987.

1980 Mathematics Subject Classification (1985 Revision). Primary 55P15, 55P60; Secondary 55P62, 55T10, 55U 10.

The first author was partially supported by the National Science Foundation. 
denotes the category of compactly generated, Hausdorff topological spaces ([21], we discuss topologies in $\S 4)$. If $A, B$ are objects in $\mathscr{C},(A, B)$ denotes the set of morphisms from $A$ to $B$. For $X, Y \in \Delta \mathscr{T}$, we make $(X, Y)$ an object in $\mathscr{T}$ by viewing it as a subspace of $\Pi\left(X_{q}, Y_{q}\right)$ where the product topology and the function space topology on $\left(X_{q}, Y_{q}\right)$ are in the sense of products and function spaces in $\mathscr{T}$ (see $\S 4$ ).

If $X \in \Delta \mathscr{T}$ and $G$ is a topological abelian group, we define the continuous cohomology groups of $X$ with coefficients in $G$ by

$$
H^{q}(X ; G)=H_{q}\left(C^{*}(X ; G), \delta\right),
$$

where $C^{q}(X ; G)$ is the set of all continuous $u: X_{q} \rightarrow G$ satisfying $u s_{i}=0$, $0 \leq i<q$, and

$$
(\delta u)(x)=\sum_{i=0}^{q+1}(-1)^{i} u\left(\partial_{i} x\right) .
$$

Here $\partial_{i}$ and $s_{i}$ are the face and degeneracy mappings of $X$. If $G$ is a topological ring, we define cup products in $C^{*}(X ; G)$ in the usual way; for $u \in C^{p}(X ; G), v \in C^{q}(X ; G), u v \in C^{p+q}(X ; G)$ is given by

$$
(u v)(x)=u\left(\partial_{p+1}^{q} x\right) v\left(\partial_{0}^{p} x\right)
$$

for $x \in X_{p+q}$. This multiplication defines a multiplication in $H^{*}(X ; G)$. In particular, if $R$ denotes the real numbers, then $H^{*}(X)=H^{*}(X ; R)$ is a graded commutative algebra. (See $\S 3$.)

Note that $H^{q}(X)$ is a topological linear space (not necessarily Hausdorff) with topology coming from $X_{q}$. If $X$ is a simplicial space with the discrete topology (that is, a simplicial set), then $H^{*}(X)$ is the ordinary, real cohomology ring of $X$.

Remark. Many authors have defined notions of continuous cohomology in various contexts. (See Mostow [16, pp. vi, 9-10] for a discussion of some of these.) In particular, Mostow [16] considers continuous cohomology theories on a category whose objects are continuous mappings $i: Y^{\prime} \rightarrow Y$ of topological spaces. His theories satisfy four axioms and, in some cases, are characterized by these axioms. The relation between Mostow's continuous cohomology and ours can be described as follows. Let $X$ be a simplicial space and let $X^{\delta}$ denote $X$ in the discrete topology. If $T^{*}$ is any continuous cohomology theory in the sense of Mostow and if each $X_{q}$ is a paracompact, then

$$
T^{*}\left(\left\|X^{\delta}\right\| \rightarrow\|X\|\right) \approx H^{*}(X)
$$

where \|\| is the fat (or unnormalized) geometric realization functor (see [19] or [16, p. 68]) and $\left\|X^{\delta}\right\| \rightarrow\|X\|$ is induced by the natural mapping $X^{\delta} \rightarrow X$. (See Corollary 7.5 of [16].)

Let $\Delta[q]$ be the simplicial standard $q$-simplex, that is, all tuples $\left(i_{0}, i_{1}, \ldots\right.$, $\left.i_{p}\right)$ where $0 \leq i_{0} \leq i_{1} \leq \cdots \leq i_{p} \leq q$. Let $e_{i}: \Delta[q-1] \rightarrow \Delta[q]$ and $d_{i}: \Delta[q+1]$ $\rightarrow \Delta[q]$ be the usual face inclusions and degeneracy projections. We form a 
simplicial Eilenberg-Mac Lane space, $K(G, n) \in \Delta \mathscr{T}$, in the usual way, namely

$$
K(G, n)_{q}=Z^{n}(\Delta[q] ; G),
$$

where $Z^{n}$ denotes normalized cocycles topologized via the topology on $G$, $\partial_{i}=e_{i}^{*}$ and $s_{i}=d_{i}^{*}$. Note that $K(G, n)$ is actually a simplicial topological abelian group.

We now define a continuous version of the Kan condition. Let $X$ be a simplicial space and $I=\left\{i_{1}, \ldots, i_{l}\right\}, 0 \leq i_{1}<i_{2}<\cdots<i_{l} \leq q, 1 \leq l \leq$ $q$. Let $X(q, I)$ be the subspace of the $l$-fold product $\left(X_{q-1}\right)^{l}$ of $l$-tuples $\left(x_{i_{1}}, \ldots, x_{i_{l}}\right)$ such that $\partial_{i} x_{j}=\partial_{j-1} x_{i}$ for $i, j \in I, i<j$, and let

$$
p_{q, I}: X_{q} \rightarrow X(q, I)
$$

be given by $p_{q, I}(x)=\left(\partial_{i_{1}} x, \ldots, \partial_{i_{l}} x\right)$. If $k \notin I$, define $\bar{s}_{k} I=\left\{j_{1}, \ldots, j_{l}\right\}$, where

$$
j_{m}= \begin{cases}i_{m} & \text { for } i_{m}<k \\ i_{m}+1 & \text { for } i_{m}>k\end{cases}
$$

Definition 2.1. A simplicial space $X$ is $K a n$ if, for each $q \geq 0$ and $I$ as above, there are mappings

$$
\lambda_{q, I}: X(q, I) \rightarrow X_{q}
$$

satisfying

(i) $\lambda_{q, I}$ are continuous,

(ii) $p_{q, I} \lambda_{q, I}=$ identity,

(iii) if $k$ or $k+1 \in I$, then, for all $x \in X_{q-1}, \lambda_{q, I} p_{q, I}\left(s_{k} x\right)=s_{k} x$,

(iv) if $k, k+1 \notin I$ and hence $I=\bar{s}_{k} I^{\prime}$, then

$$
\lambda_{q, I} p_{q, I}\left(s_{k} x\right)=s_{k} \lambda_{q-1, I^{\prime}} p_{q-1, I^{\prime}}(x) .
$$

(Conditions (iii) and (iv) assert that $\lambda_{q+1}\left(x_{i_{1}}, \ldots, x_{i_{l}}\right)$ is degenerate if the $x_{i_{j}}$ are such as to make this possible in which case the $\lambda$ 's commute with the degeneracy operators.)

Remark. It follows from Lemma 6.8 of [13] that our continuous version of the Kan condition reduces to the usual version when $X$ is a simplicial set. The definition was chosen so that if $X$ is Kan, then the simplicial function space $\mathscr{F}(Y, X)$ is Kan for any simplicial space $Y$; see Theorem 2.17 .

In $\S 6$, we show that if $Y \in \Delta \mathscr{T}$ is Kan, homotopy is an equivalence relation among maps from $X$ to $Y$ for any $X \in \Delta \mathscr{T}$. (A homotopy in $\Delta \mathscr{T}$ is a map $F: X \times \Delta[1]-Y$.) If $[X, Y]$ denotes the set of homotopy classes of maps from $X$ to $Y$, the usual arguments yield:

Theorem 2.2. The simplicial space $K(G, n)$ is Kan and there is a natural isomorphism

$$
H^{n}(X ; G) \simeq[X, K(G, n)],
$$


where the group structure on $[X, K(G, n)]$ is defined using the group structure on $K(G, n)$.

The proof of this result is given in $\S 3$. The following result makes possible our analysis of real homotopy types.

Theorem 2.3. According as $n$ is even or odd, $H^{*}(K(R, n) ; R)$ is a polynomial or an exterior algebra on one generator in dimension $n$.

Our original proof of this result, outlined in [5], made use of a version of the Van Est theorem for simplicial Lie groups. Our present proof, given in $\S 9$, uses a continuous cohomology analogue of the Serre spectral sequence of a twisted cartesian product of simplicial spaces and the Van Est theorem for $n=1$.

We next develop a de Rham approach to $H^{*}(X)$. Let $\mathscr{A}$ denote the category of differential graded, topological algebras over $R$ with unit which satisfy the following conditions: If $A \in \mathscr{A}$ and $A=\sum A^{p}$, then $A^{p}=0, p<0$, each $A^{p}$ is a locally convex Hausdorff topological vector space over $R, d: A^{p} \rightarrow A^{p+1}$, and multiplication $A^{p} \times A^{q} \rightarrow A^{p+q}$ is continuous. We denote by $\mathscr{A} \mathscr{C}$ the full subcategory of $\mathscr{A}$ consisting of those algebras which are commutative in the graded sense: $a b=(-1)^{p q} b a$, where $a \in A^{p}, b \in A^{q}$.

Following the techniques developed in [3], a central object for our study is the algebra of differential forms on the standard simplex:

$$
\Delta^{q}=\left\{\left(t_{0}, t_{1}, \ldots, t_{q}\right) \in R^{q+1}: \sum t_{i}=1, t_{i} \geq 0\right\} .
$$

There are several possible choices for these forms, all giving the same theorems, namely, differential forms with polynomial coefficients or with $C^{\infty}$ functions as coefficients. We emphasize $C^{\infty}$ functions. Let $\Omega_{q}^{p}$ be the space of $C^{\infty}$ differential $p$ forms on $\Delta^{q}$ with the $C^{\infty}$ topology. The face inclusions and degeneracy projections, $e_{i}: \Delta^{q-1} \rightarrow \Delta^{q}, d_{i}: \Delta^{q+1} \rightarrow \Delta^{q}$ induce face and degeneracy maps $\partial_{i}: \Omega_{q}^{p} \rightarrow \Omega_{q-1}^{p}$ and $s_{i}: \Omega_{q}^{p} \rightarrow \Omega_{q+1}^{p}$. Then, for fixed $p$, $\Omega^{p}=\left\{\Omega_{q}^{p}, \partial_{i}, s_{i}\right\}$ is an $\Delta \mathscr{T}$ and, for fixed $q, \Omega_{q}=\sum_{p} \Omega_{q}^{p}$ is in $\mathscr{A} \mathscr{C}$ (with differential the exterior differential). Combining these two structures, we obtain $\Omega=\left\{\Omega_{q}, \partial_{i}, s_{i}\right\}$ in $\Delta \mathscr{A} \mathscr{C}$. (Note that $\Omega_{q}^{p} \in \mathscr{T}$ because it is metrizable [21].)

Another useful gadget of this sort is the group of simplicial cochains on the standard $q$-simplex. For any topological abelian group, let $C_{q}^{p}(G)$ be defined by

$$
C_{q}^{p}(G)=C^{p}(\Delta[q]: G),
$$

the group of normalized cochains on $\Delta[q]$ with values in $G$ topologized in the usual way. Then, just as above, we obtain $C^{p}(G) \in \Delta \mathscr{T}, C_{q}(G) \in \mathscr{A}$ (using cup product as multiplication), and $C(G) \in \Delta \mathscr{A}$.

If $X \in \Delta \mathscr{T}$, let $\left\langle X, \Omega^{p}\right\rangle$ be the set of simplicial mappings, $\left(X, \Omega^{p}\right)$ topologized as a subset of the cartesian product $\Pi\left(X_{q}, \Omega_{q}^{p}\right)$, where $\left(X_{q}, \Omega_{q}^{p}\right)$ has the compact open topology. The linear structure on $\Omega^{p}$ makes $\left\langle X, \Omega^{p}\right\rangle$ into a locally convex Hausdorff space. (We have not passed to compactly generated topologies on $\left\langle X_{q}, \Omega_{q}^{p}\right\rangle$ because this might destroy the local convexity.) 
Let $\mathscr{A}(X) \in \mathscr{A} \mathscr{C}$ be defined by $\mathscr{A}^{p}(X)=\left\langle X, \Omega^{p}\right\rangle$, the space of continuous simplicial mappings from $X$ to $\Omega^{p}$. Multiplication of forms defines $\mathscr{A}(X)=\sum \mathscr{A}^{p}(X)$ as an object in $\mathscr{A} \mathscr{C}$.

An element $\omega \in \mathscr{A}^{p}(X)$ assigns to each $q$-simplex $x \in X_{q}$ a differential $p$-form on $\Delta_{q}$ and these forms fit together along faces, $e_{i}^{*} \omega(x)=\omega\left(\partial_{i} x\right)$, as well as respecting degeneracy. We think of $\mathscr{A}(X) \in \mathscr{A} \mathscr{C}$ as the algebra of differential forms on $X$.

In a completely analogous fashion we set

$$
C^{q}(X ; G)=\left\langle X, C^{q}(G)\right\rangle
$$

and obtain $C(X ; G) \in \mathscr{A}$. It is easy to see that this definition of $C(X ; G)$ agrees with our earlier definition. (See Lemma 3.5.)

In $\S 5$ we define a simplicial map $\Psi: \Omega \rightarrow C(R)$ and composition with $\Psi$ gives a map

$$
\Psi: \mathscr{A}(X) \rightarrow C^{*}(X)=C^{*}(X ; R) .
$$

This map is the usual map which, for $\omega \in \mathscr{A}^{p}(X)$ and $x \in X_{p}$, is given by

$$
\Psi(\omega)(x)=\int_{\Delta^{p}} \omega(x) \text {. }
$$

We define the continuous de Rham cohomology of $X$ by $H_{d R}^{q}(X)=H_{q}(\mathscr{A}(X))$.

In $\S 5$ we prove

Theorem 2.4. The map $\Psi$ induces a ring isomorphism $\Psi: H_{d R}^{*}(X) \rightarrow H^{*}(X)$.

We next describe a simplicial space $\Delta(A)$ for an algebra $A \in \mathscr{A} \mathscr{C}$ which has the property that if $A$ is free, nilpotent, and of finite type (see definition below), then

$$
H_{*}(A) \approx H_{*}(\mathscr{A}(\Delta(A))) .
$$

If $A, B \in \mathscr{A} \mathscr{C}$, we form $A \otimes B \in \mathscr{A} \mathscr{C}$ algebraically in the usual way and with the projective topology on $A_{p} \otimes B_{q}$ ([23]; the projective topology is the strongest topology making $A_{p} \otimes B_{q}$ into a locally convex topological vector space such that $A_{p} \times B_{q}-A_{p} \otimes B_{q}$ is continuous).

If $A, B \in \mathscr{A}$ we topologize $(A, B)$ so it is in $\mathscr{T}$ as follows: For a topological space $U$ let $k(U)$ be $U$ with its compactly generated topology (see $\S 4)$. For $U$ and $V$ in $\mathscr{T}$ their $\mathscr{T}$ product is $k$ of their cartesian product and $(U, V)$ is topologized by $k$ of the compact open topology. Then, working in $\mathscr{T},(A, B)$ is topologized as a subspace of $\Pi\left(A_{p}, B_{p}\right)$.

In general, for $\Gamma \in \Delta \mathscr{A}, X \in \Delta \mathscr{T}$ and $A \in \mathscr{A}$, we form $\Gamma \otimes A \in \Delta \mathscr{A}$, $\langle X, \Gamma\rangle \in \mathscr{A}$ and $(A, \Gamma) \in \Delta \mathscr{T}$ by

$$
(\Gamma \otimes A)_{q}=\Gamma_{q} \otimes A, \quad\langle X, \Gamma\rangle^{p}=\left\langle X, \Gamma^{p}\right\rangle, \quad(A, \Gamma)_{q}=\left(A, \Gamma_{q}\right) .
$$

For $A \in \mathscr{A} \mathscr{C}$ we define its real simplicial form by $\Delta(A)=(A, \Omega)$. For example, if $A=R[x]$ is the polynomial or exterior algebra on a generator $x$ in dimension $n$ with $d x=0$, then

$$
\Delta(R[x])_{q}=\left\{\omega \in \Omega_{q}^{n}: d \omega=0\right\}
$$


which, as we will see in $\S 9$, is a differential form model of $K(R, n)$. If $A=$ $R[x, y], d x=0$, and $d y=x^{k}$, then

$$
\Delta(A)_{q}=\left\{(u, v) \in \Omega_{q}^{n} \times \Omega_{q}^{k n-1}: d u=0, d v=u^{k n}\right\}
$$

is a two-stage Postnikov system.

Note that $\Delta: \mathscr{A} \mathscr{C} \rightarrow \Delta \mathscr{T}$ and $\mathscr{A}: \Delta \mathscr{T} \rightarrow \mathscr{A} \mathscr{C}$ are contravariant functors.

We next define the notion of an algebra $A \in \mathscr{A} \mathscr{C}$ being FNF, that is free, nilpotent, and of finite type. If $A \in \mathscr{A} \mathscr{C}, a \in A^{n+1}$ and $d a=0$, we define $A[x]=A_{a}[x] \in \mathscr{A} \mathscr{C}$ to be $A \otimes R[x]$ as a graded topological algebra, $\operatorname{dim} x=n$, and we define the differential by

$$
d(b \otimes x)=d b \otimes x+(-1)^{p}(b a \otimes 1)
$$

for $b \in A^{p}$.

Definition 2.5. An algebra $A \in \mathscr{A} \mathscr{C}$ is FNF (free, nilpotent, and of finite type) if it is isomorphic to $\bigcup A_{n}$ where $A_{0}=R$ and $A_{n}=A_{n-1}[x]$. We also require that for any $m$, there is an $n$ such that $A^{m}=A_{n}^{m}$. We say that $A$ is minimal if, in addition, $d a$ is decomposable for all $a \in A$. An algebra $B \in \mathscr{A} \mathscr{C}$ is NF ( $R$-nilpotent and of finite type) if there is an FNF algebra $A$ and $f: A \rightarrow B$ inducing an isomorphism on homology.

The proof of the next result is straightforward and left to the reader (see, for example, [3, Proposition 7.4]).

Theorem 2.6. If $A \in \mathscr{A} \mathscr{C}$ is $R$-nilpotent and of finite type and if $H_{0}(A) \simeq R$, then there is a minimal algebra $M$ and $f: M \rightarrow A$ such that $f_{*}: H_{*}(M) \rightarrow$ $H_{*}(A)$ is an isomorphism. If $f^{\prime}: M^{\prime} \rightarrow A$ is another such pair, there is an isomorphism $h: M \rightarrow M^{\prime}$ such that $f^{\prime} h$ and $f$ are homotopic (see below for the definition of homotopy in $\mathscr{A}$ ). If $A \in \mathscr{A} \mathscr{C}$ is of finite type, $H_{0}(A) \simeq R$, and $H_{1}(A) \simeq 0$, then $A$ is $R$-nilpotent.

For any $A \in \mathscr{A} \mathscr{C}$, we have a map $i: A \rightarrow \mathscr{A}(\Delta(A))$ defined by $i(a)(u)=$ $u(a)$. In $\S 4$ we prove

Lemma 2.7. If for each $p, A^{p}$ has compactly generated topology (for example, if it is metrizable) then $i: A \rightarrow \mathscr{A}(\Delta(A))$ is continuous.

In $\S 9$ we use the Serre spectral sequence for continuous cohomology to prove

Proposition 2.8. If $A$ is FNF, then $i_{*}: H_{q}(A) \rightarrow H_{q}(\mathscr{A}(\Delta(A)))$ is an isomorphism for all $q \geq 0$.

For any simplicial space $X$, define $j: X \rightarrow \Delta \mathscr{A}(X)$ by the formula

$$
j(x)(w)=w(x)
$$


for $x \in X, w \in \mathscr{A}(X)=(X, \Omega)$. In $\S 4$ we prove

Lemma 2.10. The mapping $j: X \rightarrow \Delta \mathscr{A}(X)$ defined above is continuous.

Theorem 2.11. If $\mathscr{A}(X)$ is connected, R-nilpotent and of finite type, there is a minimal algebra $M$ and $f: X \rightarrow \Delta(M)$ such that

$$
f^{*}: H^{*}(\Delta(M)) \rightarrow H^{*}(X)
$$

is an isomorphism. Furthermore, if $M^{\prime}$ and $f^{\prime}$ are another such pair, there is an isomorphism $h: M \rightarrow M^{\prime}$ such that $f \Delta(h)$ and $f^{\prime}$ are homotopic.

Since the proof is short we include it here. Suppose $X$ is $R$-nilpotent and of finite type. By Theorem 2.6 there is a minimal algebra $M$ and a map $g: M \rightarrow$ $\mathscr{A}(X)$ such that $g$ induces an isomorphism on $H_{*}$. Let $f: X \rightarrow \Delta(M)$ be the composite:

$$
X \stackrel{j}{\rightarrow} \Delta(\mathscr{A}(X)) \stackrel{\Delta(g)}{\rightarrow} \Delta(M) .
$$

By Theorem 2.4 it is sufficient to show that $\mathscr{A}(f): \mathscr{A}(\Delta(M)) \rightarrow \mathscr{A}(X)$ induces an isomorphism on homology. Consider the commutative diagram

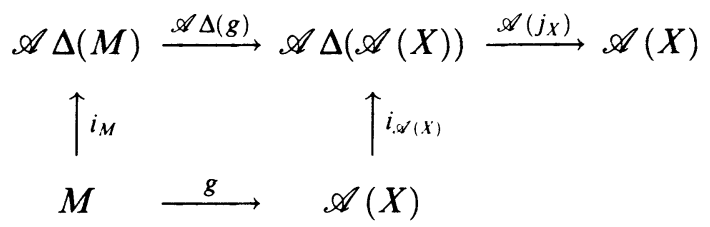

One easily checks that $\mathscr{A}\left(j_{X}\right) i_{\mathscr{A}(X)}$ is the identity. By Proposition $2.8, i_{M}$ induces an isomorphism in continuous cohomology as does $g$ by construction. Hence $\mathscr{A}\left(j_{X} \Delta(g)\right)=\mathscr{A}(f)$ induces an isomorphism in continuous cohomology. The last part of Theorem 2.11 follows from Theorem 2.6 and the fact that $\Delta$ preserves homotopy (see Theorem 2.19 below).

The relation between the homotopy types of $X$ and $\Delta(M)$ in $\Delta \mathscr{T}$ and the relation between their associated simplicial sets (forgetting the topology) is at present unclear to the authors. For example, it is probably true that $|\Delta(M)|$ is contractible. We next give very stringent hypotheses on $X$ which will ensure that $X$ and $\Delta(M)$ are related as one would most optimistically expect. (See Theorem 2.13.)

Suppose $X \in \Delta \mathscr{T}$ is $\mathrm{Kan}, x_{0} \in X$ is a base point and $\pi_{n}\left(X, x_{0}\right)$ denotes the usual homotopy groups of $X$ as a simplicial set, with the topology coming from the topology on $X_{n} ; \pi_{n}\left(X, x_{0}\right)$ is a quotient of

$$
\left\{x \in X_{n}: \partial_{i} x=x_{0}, 0 \leq i \leq n\right\}
$$

with the quotient topology. Let

$$
\pi^{n}\left(X, x_{0}\right)=\text { Hom }_{\text {cont }}\left(\pi_{n}\left(X, x_{0}\right), R\right) .
$$

Suppose $\pi$ is a topological abelian group, $X \in \Delta \mathscr{T}, u \in C^{n+1}(X, \pi)$, and $\delta u=0$. Let $p: E_{u} \rightarrow X$ be the fibration (see $\S 6$ )

$$
E(u)_{q}=\left\{(x, v) \in X_{q} \times C^{n}(\Delta[q] ; G) \mid t_{x}^{*} u=\delta v\right\}
$$


where $p(x, v)=x$, and $t_{x}: \Delta[q] \rightarrow X$ is the unique map such that $t_{x}(0, \ldots, q)$ $=x$.

Definition 2.12. The simplicial space $X$ has a simple Postnikov system if it has the homotopy type of $X^{\prime}$ in $\Delta \mathscr{F}$ and $X^{\prime}=\lim _{\leftarrow} X_{n}, X_{n}=E\left(u_{n}\right), u_{n} \in$ $C^{n+1}\left(X_{n-1} ; \pi_{n}\right)$, where $\pi_{n}$ is a topological abelian group and $X_{0}=\mathrm{pt}$. If in addition, each $\pi_{n}$ is locally Euclidean we say that $X$ is of finite type. Note that $\pi_{n}\left(X^{\prime}\right) \approx \pi_{n}$ as topological groups.

In $\S 10$ we prove

Theorem 2.13. If $X$ has a simple Postnikov system and is of finite type, and if $f: X \rightarrow \Delta(M)$ is as in Theorem 2.11 , then

$$
f^{*}: \pi^{i}(\Delta(M)) \approx \pi^{i}(X) .
$$

Furthermore, $\pi^{i}(\Delta(M)) \approx(M / \hat{M})^{i}$ where $\hat{M} \subset M$ is the ideal of decomposable elements.

Remark. If $X \in \Delta \mathscr{S}$ is simple and of finite type, then, viewed as an object in $\Delta \mathscr{T}$ with the discrete topology, it has a simple Postnikov system and is of finite type.

We next investigate how maps behave under the functor $\Delta$. Suppose $A, B \in$ $\mathscr{A} \mathscr{C}$. Following [3], we define a function space $\mathscr{F}(A, B) \in \Delta \mathscr{T}$ by

$$
\mathscr{F}(A, B)=(A, \Omega \otimes B) .
$$

Thus a $q$-simplex in $\mathscr{F}(A, B)$ is a DGA map $A \rightarrow \Omega_{q} \otimes b$ and the face and degeneracy mappings are defined in terms of those for $\Omega$. Note that $\Delta(A)=$ $\mathscr{F}(A, R)$. As usual, we define $\mathscr{F}(X, Y) \in \Delta \mathscr{T}$, for $X, Y \in \Delta \mathscr{T}$ by

$$
\mathscr{F}(X, Y)_{q}=(\Delta[q] \times X, Y) .
$$

Let composition

$$
\mathscr{O}: \mathscr{F}(A, B) \times \mathscr{F}(B, C) \rightarrow \mathscr{F}(A, C)
$$

be defined by

$\left(A, \Omega_{q} \otimes B\right) \times\left(B, D_{q} \otimes C\right) \stackrel{\text { id } \times b}{\rightarrow}\left(A, \Omega_{q} \otimes B\right) \times\left(\Omega_{q} \otimes B, \Omega_{q} \otimes C\right) \stackrel{\mathscr{O}_{0}}{\rightarrow}\left(A, \Omega_{q} \otimes C\right)$, where $\mathscr{O}_{0}$ is the usual composition and $b(u)(\omega \otimes b)=(\omega \otimes 1)(u(b))$.

Taking $C=R$ in (2.14) and taking the adjoint of $\mathscr{O}$, we obtain a map

$$
\Delta: \mathscr{F}(A, B) \rightarrow \mathscr{F}(\Delta(B), \Delta(A))
$$

which extends the map $\Delta:(A, B) \rightarrow(\Delta(B), \Delta(A))$.

Let $\gamma: \mathscr{F}(A, \mathscr{A}(X)) \rightarrow \mathscr{F}(X, \Delta(A))$ be the adjoint of

$$
\mathscr{F}(A, \mathscr{A}(X)) \times X \stackrel{1 \times j}{\rightarrow} \mathscr{F}(A, \mathscr{A}(X)) \times \mathscr{F}(\mathscr{A}(X), R) \stackrel{\mathscr{O}}{\rightarrow} \mathscr{F}(A, R) .
$$

In Lemma 4.4 we prove that $\Delta$ and $\gamma$ are continuous for all $q$ when $B^{q}$ is finite dimensional. The proof of these results uses the fact that we are working with compactly generated spaces. One of our main results is that $\Delta$ and $\gamma$ are weak equivalences when $A$ and $B$ are FNF and $X$ is connected. 
Definition 2.16. If $X, Y$ in $\Delta \mathscr{F}$ are Kan, then $f: X \rightarrow Y$ is a weak equivalence if for all base points $x_{0} \subset X$ and all $n \geq 0, f_{*}: \pi_{n}\left(X, x_{0}\right) \rightarrow \pi_{n}\left(Y, f\left(x_{0}\right)\right)$ is a group isomorphism or set isomorphism when $n=0$. ( $f_{*}$ is automatically continuous but need not be a homeomorphism.)

Remark. We note that a weak equivalence $f: X \rightarrow Y$ need not induce isomorphisms on continuous cohomology. For example, the "identity" mapping from $K\left(R^{\delta}, n\right)$ onto $K(R, n)$ is certainly a weak equivalence but is not a continuous-cohomology isomorphism. However, if $X$ and $Y$ have simple Postnikov systems and $f: X \rightarrow Y$ is a weak equivalence with the property that each $f_{q}: \pi_{q}\left(X, x_{0}\right) \rightarrow \pi_{q}\left(Y, f\left(x_{0}\right)\right)$ is a homeomorphism, then $f$ can be shown to induce isomorphisms continuous cohomology.

In $\S 6$ we prove the following two results:

Theorem 2.17. If $X, Y \in \Delta \mathscr{T}$ and $Y$ is Kan, then $\mathscr{F}(X, Y)$ is Kan.

Theorem 2.18. If $A, B \in \mathscr{A} \mathscr{C}$ and $A$ is $F N F$, then $\mathscr{F}(A, B)$ and $\Delta(A)$ are Kan.

The usual arguments and Theorem 2.17 show that homotopy for maps $f: X$ $\rightarrow Y$ is an equivalence relation when $Y$ is Kan and $[X, Y]=\pi_{0}(\mathscr{F}(X, Y))$. For $A, B \in \mathscr{A} \mathscr{C}$, define a homotopy to be a map $F: A \rightarrow \Omega_{1} \otimes B$. Then Theorem 2.18 yields the fact that homotopy is an equivalence relation in $\mathscr{A}$ and $[A, B]=\pi_{0}(\mathscr{F}(A, B))$. Since $\Delta$ maps function spaces to function spaces we have

Lemma 2.19. The functor $\Delta$ induces a map $[A, B] \rightarrow[\Delta(B), \Delta(A)]$ when $A$ is FNF.

In $\S 7$ we prove

Theorem 2.20. If $A$ and $B \in \mathscr{A} \mathscr{C}$ are FNF then

$$
\Delta: \mathscr{F}(A, B) \rightarrow \mathscr{F}(\Delta(B), \Delta(A))
$$

is a weak equivalence. In particular, $\Delta$ induces a bijection $[A, B] \simeq[\Delta(B), \Delta(A)]$. If, in addition $X \in \Delta \mathscr{T}$ is 0 -connected $\left(\pi_{0}(X)=\mathrm{pt}\right)$, then

$$
\gamma: \mathscr{F}(A, \mathscr{A}(X)) \rightarrow \mathscr{F}(X, \Delta(A))
$$

is a weak equivalence. If $A \in \mathscr{A} \mathscr{C}$ is FNF and $B, C, f: B \rightarrow C$ are in $\mathscr{A} \mathscr{C}$ with $f^{*}: H_{*}(B) \simeq H_{*}(C)$, then the induced map $\mathscr{F}(A, B) \rightarrow \mathscr{F}(A, C)$ is a weak equivalence.

We can recast some of our results into a categorical form as in Quillen [17] as follows: Let $\mathscr{A} \mathscr{C}_{0}$ be the full subcategory of $\mathscr{A} \mathscr{C}$ consisting of objects which are NF. Let $\Delta \mathscr{F}_{0}$ be the full subcategory of $\Delta \mathscr{F}$ consisting of those $X$ such that $\hat{\mathscr{A}}(X)$ is FNF. 
Definition 2.21. A mapping $h: A \rightarrow B$ in $\mathscr{A} \mathscr{C}_{0}$ is an $R$-equivalence if $h_{*}: H_{*}(A)$ $\approx H_{*}(B)$. A mapping $f: X \rightarrow Y$ in $\Delta \mathscr{T}_{0}$ is an $R$-equivalence if $f^{*}: H^{*}(Y) \approx$ $H^{*}(X)$.

Let $\Delta \mathscr{T}_{0 R}$ and $\mathscr{A} \mathscr{C}_{0 R}$ be the categories $\Delta \mathscr{T}_{0}$ and $\mathscr{A} \mathscr{C}_{0}$ localized with respect to their $R$-equivalences [17]. We define a functor $\Delta_{h}: \mathscr{A}_{\mathscr{C}_{0 R}} \rightarrow \Delta \mathscr{T}_{0 R}$ as follows: For each $A \in \mathscr{A} \mathscr{C}_{0}$ choose $f_{A}: M_{A} \rightarrow A$, where $M$ is FNF and $f_{A}$ is an $R$-equivalence. Let $\Delta_{h}(A)=\Delta\left(M_{A}\right)$. By Theorem 2.20, if $A$ is FNF and $g: B \rightarrow C$ is an $R$-equivalence, $g$ induces an isomorphism $[A, B] \rightarrow[A, C]$. Hence, if $g: B \rightarrow C$ is a map, there is a unique map $\bar{g}: M_{B} \rightarrow M_{C}$, in $\mathscr{A}_{0 R}$ (localizing makes homotopic maps equal) such that $f_{C} \bar{g}=g f_{B}$. Let $\Delta_{h}(g)=\bar{g}$. Then Theorems 2.11 and 2.20 immediately give

Theorem 2.22. The functor $\Delta_{h}: \mathscr{A}_{\mathscr{C}_{0 R}} \rightarrow \Delta \mathscr{R}_{0 R}$ is an equivalence of categories in the sense that $\Delta_{h}$ is an isomorphism on morphism sets and each object of $\Delta \mathscr{R}_{0 R}$ is isomorphic to $\Delta_{h}(M)$ for some $M$.

Remark. A casual reading of Theorem 2.22 might suggest that if $A, B \in \mathscr{A} \mathscr{C}_{0}$ and $f: A \rightarrow B$ induces an isomorphism on $H_{*}$, then $\Delta(f): \Delta(B) \rightarrow \Delta(A)$ induces an isomorphism on $H^{*}$. If $A$ and $B$ are not free this may not be true. It is true that if $f$ is a homotopy equivalence, i.e. there is a map $g: B \rightarrow A$ such that $f g$ and $g f$ are homotopic to the identity, then $\Delta(f)$ is a homotopy equivalence because $\Delta$ extends to $\mathscr{F}(A, B) \rightarrow \mathscr{F}(\Delta(B), \Delta(A))$.

We remark that if we replace $R$ by $Q$, smooth forms by rational polynomial forms, and simplicial spaces by simplicial sets throughout this paper, then our proofs establish a somewhat extended version of the Quillen-Sullivan Rational Homotopy Theory $[17,26]$. (See Theorems 2.11 and 2.20.)

Let $\alpha: \Delta \mathscr{S} \rightarrow \Delta \mathscr{T}$ be the functor which assigns to each simplicial set $X$, the simplicial space $X$ with the discrete topology. One may localize with respect to the rationals to form $\Delta \mathscr{S}_{0 Q}$, exactly as above and $\sigma$ induces a functor $\bar{\alpha}: \Delta \mathscr{S}_{0 Q} \rightarrow \Delta \mathscr{T}_{0 R}$.

Our next result (proved in $\S 11$ ) shows that this functor is neither injective nor surjective.

Theorem 2.23. There are simply connected simplicial sets $X_{1}$ and $X_{2}$ each of finite type such that $X_{1}$ and $X_{2}$ are not isomorphic in $\Delta \mathscr{S}_{0 Q}$ but $\bar{\alpha}\left(X_{1}\right)$ and $\bar{\alpha}\left(X_{2}\right)$ are isomorphic in $\Delta \mathscr{T}_{0 R}$. In addition, there is an FNF $A \in \mathscr{A} \mathscr{C}$ such that $\Delta(A)$ is not isomorphic to anything in the image of $\bar{\alpha}$.

We next give a formulation of the main result of [6] in our context.

If $B$ is a commutative graded algebra of finite type, we view it as being in $\mathscr{A} \mathscr{C}$ with zero differential. Let $M(B)$ be a minimal algebra and $\gamma: M(B) \rightarrow B$ a map such that $\gamma$ induces an isomorphism

$$
H_{*}(M(B)) \rightarrow H_{*}(B)=B .
$$

Definition 2.24. A simplicial space $X \in \Delta \mathscr{T}$ is $R$-formal if there is a map $g: X \rightarrow \Delta\left(M\left(H^{*}(X)\right)\right.$ inducing an isomorphism in cohomology. 
Theorem 2.25. If $N$ is a compact nilpotent Kähler manifold, then the total singular complex $\Delta(N)$ in the discrete topology is R-formal. Hence, there is a minimal algebra $M\left(H^{*}(N)\right) \in \mathscr{A} \mathscr{C}$ and a map $g: \Delta(N) \rightarrow \Delta\left(M\left(H^{*}(N)\right)\right)$ inducing isomorphisms on $H^{*}$ and on $\pi^{*}$.

We conclude this section with some examples of simplicial spaces.

Example 1. Let $W$ be a topological space and let $\Delta(W)$ be the simplicial space of singular simplices of $W$ with the compact-open topology. Graeme Segal has proved (unpublished) that if $W$ is a $\mathrm{CW}$ complex, then

$$
H^{*}(\Delta(W) ; G) \simeq H^{*}\left(\Delta(W)^{\delta} ; G\right)
$$

for any $G$. Thus the continuous cohomology of $\Delta(W)$ is isomorphic to the singular cohomology of $W$.

Example 2. For any topological Lie group, we have defined $K(G, 1)$ by

$$
K(G, 1)_{q}=Z^{1}(\Delta[q], G)
$$

Equivalently, $K(G, 1)$ is the Bar Construction on $G$. If $G$ is a Lie group, the Van Est theorem [24] expresses $H^{*}(K(G, 1))$ in terms of cohomology of the Lie algebra of $G$. These results can be extended to the case where $G$ is a simplicial Lie group [5].

We note that the geometric realization of $K(G, 1)$ is essentially Milnor's construction [15] of the classifying space $B G$ of $G$. (See [18, p. 107].)

Example 3. The previous example is a special case of the nerve of a topological category. Recall that a topological category is a category $\mathscr{C}$ in which both $\operatorname{Obj}(\mathscr{C})$ and $\operatorname{Morph}(\mathscr{C})$ are topological spaces and all structure mappings are continuous. The nerve of $\mathscr{C}$ is the simplicial space $N(\mathscr{C})$ with $N(\mathscr{C})_{0}=$ $\operatorname{Obj}(\mathscr{C})$ and $N(\mathscr{C})_{q}=$ all sequences of $q$-composable morphisms, $q>0$. The face and degeneracy mappings are defined in the usual way. With this definition, $K(G, 1)$ is the nerve of the category $\mathscr{G}$ with one object, $\operatorname{Morph}(\mathscr{G})=G$ and with multiplication as composition.

Example 4. Let $\zeta_{q} \rightarrow B G L_{q}$ be the universal real $q$-plane bundle. Let $B(q)$ be the simplicial set consisting of pairs $(T, \mathscr{F})$, where $T: \Delta^{p} \rightarrow B G L_{q}$ is smooth and $\mathscr{F}$ is a smooth foliation on $T^{*} \zeta_{q}$ transverse to fibres. For fixed $T$, it is easy to define a sensible topology on $\{(T, \mathscr{F})\}$ making $B(q)$ into a simplicial space. Then, the geometric realization $\left|B(q)^{\delta}\right|$ is a model for $B \Gamma_{q}$ and $H^{*}(B(q))$ is a plausible definition of the continuous cohomology of $B \Gamma_{q}$.

Let $\overline{B(q)}$ be the fibre of the natural mapping $B(q) \rightarrow \Delta\left(B G L_{q}\right)^{\delta}, L_{q}$ be the Lie algebra of $C^{\infty}$ vector fields on $R^{q}$ in the $C^{\infty}$ topology, and let $C^{*}\left(L_{q}\right) \in$ $\mathscr{A} \mathscr{C}$ be the algebra of continuous cochains on $L_{q}$ (continuous skew forms on $L_{q}$ with differential defined by Lie bracket). Then $\overline{B \Gamma}_{q}=\left|\overline{B(q)}^{\delta}\right|$ is the 
classifying space for foliations with trivialized normal bundle. In [11], Haefliger shows that $\overline{B(q)}=\Delta\left(C^{*}\left(L_{q}\right)\right)$ and

$$
C^{*}\left(L_{q}\right) \rightarrow \mathscr{A}\left(\Delta\left(C^{*}\left(L_{q}\right)\right)\right)
$$

gives the characteristic map

$$
H^{*}\left(L_{q}\right) \rightarrow H^{*}\left(\overline{B \Gamma_{q}}\right) .
$$

A similar construction can be used to obtain a map

$$
H^{*}\left(L_{q}, O_{q}\right) \rightarrow H^{*}\left(B \Gamma_{q}\right) .
$$

Example 5. Let $\Gamma_{q}$ be the topological category with $\operatorname{Obj}\left(\Gamma_{q}\right)=R^{q}$ and morphisms all germs of local diffeomorphisms of $R^{q}$ in the sheaf topology. Then the nerve of $\Gamma_{q}, N\left(\Gamma_{q}\right)$, is a simplicial manifold with each $N\left(\Gamma_{q}\right)_{k}$ a smooth manifold of dimension $q$ which is neither Hausdorff nor paracompact. The geometric realization of $N \Gamma_{q}$ as a simplicial space is essentially a Haefliger classifying space $B \Gamma_{q}$ for $\Gamma_{q}$ structures [9, §5]. In fact, Haefliger [8] and Thurston [22] have proved that $B \Gamma_{q}$ also "classifies" foliations of codimension $q$.

The continuous cohomology of $N \Gamma_{q}$ is unknown to the authors. Haefliger [11] has given a somewhat complicated definition of smooth cohomology in this case.

\section{SOME PROPERTIES OF CONTINUOUS COHOMOLOGY}

In this section, we derive some of the elementary properties of the continuous cohomology functor and show it to be representable. We also prove that any simplicial topological group is Kan.

By a mapping $f: X \rightarrow Y$ between simplicial spaces, we will always mean a continuous simplicial mapping, that is a sequence $f_{q}: X_{q} \rightarrow Y_{q}, q=0,1, \ldots$, of continuous mappings which commute with face and degeneracy operators. Clearly such a mapping defines a sequence of homomorphisms $f^{q}: H^{q}(Y ; G) \rightarrow$ $H^{q}(X ; G)$ making $H^{q}(; G)$ a contravariant functor from the category of simplicial spaces to the category of topological groups.

Let $X$ and $Y$ be simplicial spaces and $f, g: X \rightarrow Y$ mappings. As indicated earlier, a homotopy from $f$ to $g$ is a mapping $F: X \times \Delta[1] \rightarrow Y$ with $F\left(x, s_{0}^{q} 0\right)=f(x), F\left(x, s_{0}^{q} 1\right)=g(x)$ for $x \in X_{q}$, all $q$. Here $X \times \Delta[1]$ is the product of simplicial spaces, $\Delta[1]$ having the discrete topology. As usual, we say $f$ is homotopic to $g$ if there is a homotopy from $f$ to $g$ and we write $f \simeq g$.

In general, the relation $f \simeq g$ is not an equivalence relation. For simplicial sets, it is sufficient for $Y$ to satisfy the Kan condition. In our context, we need to require that $Y$ satisfy our continuous version of the Kan condition (Definition 2.1). 
Proposition 3.1. Let $X$ and $Y$ be simplicial spaces and assume that $Y$ satisfies the continuous Kan condition. Then the relation $f \simeq g$ for maps $f, g: X \rightarrow Y$ is an equivalence relation.

This result is an immediate consequence of Theorem 6.2 (see Corollary 6.4). We show next that continuous cohomology is a homotopy functor.

Proposition 3.2. Let $f, g: X \rightarrow Y$ be homotopic mappings between the simplicial spaces $X$ and $Y$. Then

$$
f^{*}=g^{*}: H^{*}(Y ; G) \rightarrow H^{*}(X ; G) .
$$

Proof. Let $f: X \times \Delta[1] \rightarrow Y$ be a homotopy from $f$ to $g$. For each $q \geq 0$, define continuous mappings $h_{i}: X_{q} \rightarrow Y_{q+1}, 0 \leq i \leq q$, by

$$
h_{i}(x)=F\left(s_{i} x, s_{q} \cdots s_{i+1} s_{i-1} \cdots s_{0} \sigma_{1}\right) \text {, }
$$

where $\sigma_{1}=(0,1)$ is the nondegenerate one simplex in $\Delta[1]_{1}$. Define

$$
D: C^{q+1}(Y) \rightarrow C^{q}(X)
$$

by

$$
D(u)=\sum_{i=0}^{q}(-1)^{i} u h_{i}
$$

One checks easily that $\delta D+D \delta=f^{*}-g^{*}$.

In the previous section, we defined a multiplication in $C^{*}(X ; G)$ for $G$ a topological ring by

$$
(u v)(x)=u\left(\partial_{p+1}^{q} x\right) v\left(\partial_{0}^{p} X\right)
$$

where $u \in C^{p}(X ; G), v \in C^{q}(X ; G)$, and $x \in X_{p+q}$. An easy computation proves that

$$
\delta(u v)=(\delta u) v+(-1)^{p} u \delta v
$$

so that a multiplication is defined in $H^{*}(X ; G)$. Our next result gives the basic properties of this multiplication.

Proposition 3.3. The multiplication on $H^{*}(X ; G)$ defined above is associative, graded commutative, and has a unit. Furthermore, if $f: X \rightarrow Y$ is a mapping of simplicial spaces, then $f^{*}: H^{*}(Y ; G) \rightarrow H^{*}(X ; G)$ is a homomorphism of graded rings.

Proof. If $1 \in C^{0}(X ; G)$ is the cocycle corresponding to the constant 1 mapping $X_{0} \rightarrow R$, then each of the assertions of Proposition 3.3, except for the commutativity, holds on the cochain level. To prove commutativity, let $u \in Z^{p}(X ; G)$, $v \in Z^{q}(X ; G)$, and define the "cup one" product $u \cup_{1} v \in C^{p+q-1}(X ; G)$ by

$$
\left(u \cup_{1} v\right)(x)=\sum_{j=0}^{p-1}(-1)^{(p-j)(q+1)} u\left(\partial_{j+1}^{q-1} x\right) v\left(\partial_{0}^{j} \partial_{j+q+1}^{p-j-1} x\right) .
$$

Then

$$
\delta\left((-1)^{p+q} u \cup_{1} v\right)=u v+(-1)^{p q} v u
$$

(See Steenrod [20, Theorem 5.1].) 
Let $G$ be a topological abelian group and let $l_{n} \in H^{n}(K(G, n) ; G)$ be the class represented by the cocycle $l_{n} \in C^{n}(K(G, n) ; G)$ defined by $l_{n}(u)=u\left(\sigma_{n}\right)$, where $u \in K(G, n)_{n} \in Z^{n}(\Delta[n] ; G)$ and $\sigma_{n}$ is the nondegenerate element of $\Delta[n]_{n}$.

Proposition 3.4. For any simplicial space $X$ and $n>0$, the assignment $f \mapsto$ $f^{*}{ }_{l_{n}}$ defines a natural isomorphism from $[X, K(G, n)]$ onto $H^{n}(X ; G)$.

Here $[X, Y]$ denotes the set of homotopy classes of mappings from $X$ to $Y$. Note that homotopy is an equivalence relation in this case since $K(G, n)$ is a simplicial topological group (see Proposition 3.8 below).

Proof. Recall that in $\S 2$ we defined a simplicial abelian group $C^{n}(G)$ by $C^{n}(G)_{q}$ $=C^{n}(\Delta[q] ; G)$. For any simplicial space $X$, let $f \mapsto \hat{f}$ be the homomorphism from $\left(X, C^{n}(G)\right)$ into $C^{n}(X ; G)$ defined by $\hat{f}(x)=f_{n}(x)\left(\sigma_{n}\right)$, where $\sigma_{n}=$ $(0,1, \ldots, n) \in \Delta[n]_{n}$. Proposition 3.4 is an easy consequence of the following two lemmas.

Lemma 3.5. The mapping $f \mapsto \hat{f}$ is an isomorphism and the diagram

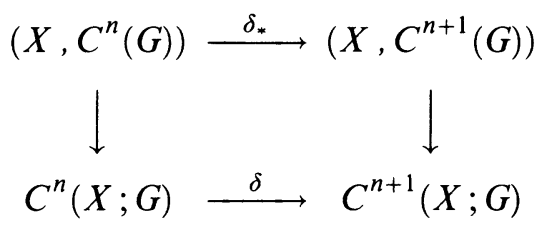

is commutative (where $\delta_{*}$ is induced by $\delta: C^{n}(G) \rightarrow C^{n+1}(G)$ ).

Proof. Note that, for $f \in\left(X, C^{n}(G)\right)$,

$$
f_{q}(x)\left(i_{0}, \ldots, i_{n}\right)=f_{q}(x)\left(\varepsilon_{J} \sigma_{n}\right)=\left(\partial_{J} f_{q}(x)\right)\left(\sigma_{n}\right)=f_{n}\left(\partial_{J} x\right)\left(\sigma_{n}\right),
$$

where $J=\left(j_{1}, \ldots, j_{q-n}\right)$ is the complement to $\left(i_{0}, \ldots, i_{n}\right)$ in $\{0,1, \ldots, q\}$. In fact, given $u: X_{n} \rightarrow G$, if we define $f_{q}: X_{q} \rightarrow C^{n}(G)_{q}$ by

$$
f_{q}(x)\left(i_{0}, \ldots, i_{n}\right)=u\left(\partial_{J} x\right) \text {, }
$$

then $f=\left\{f_{q}\right\}$ is a simplicial mapping with $\hat{f}=u$. It follows that $f \mapsto \hat{f}$ is bijective. The proof that diagram (3.6) is commutative is straightforward.

Lemma 3.7. Let $f$ and $g$ be elements of $\left(X, C^{n}(G)\right)$. Then $f \simeq g$ if and only if $\hat{f}-\hat{g}=\delta h$ for some $h \in C^{n-1}(X ; G)$.

Proof. We can assume that $g=0$. If $F: X \times \Delta[1] \rightarrow C^{n}(G)$ is a homotopy between $f$ and the zero mapping, define $h_{i}: X_{q} \rightarrow C^{n}(G)_{q+1}$ as in the proof of Proposition 3.2. Let $h \in C^{n-1}(X ; G)$ be defined by

$$
h(x)=\sum_{i=0}^{n}(-1)^{i} h_{i}(x)\left(\sigma_{n}\right) \text {. }
$$

Then $\delta h=f$. 
Conversely, if $\hat{f}=\delta h$, we define $H: X_{n} \times \Delta[1]_{n} \rightarrow G$ by

$$
\begin{gathered}
H\left(x, s_{n-1} \cdots s_{j+1} s_{j-1} \cdots s_{0} \sigma\right)=0 \quad \text { if } 0 \leq j<n-1 \\
H\left(x, s_{n-2} \cdots s_{0} \sigma\right)=(-1)^{n} h\left(\partial_{n} x\right) \quad \text { if } j=n-1 \\
H\left(x, s_{n-1} \cdots s_{0} 1\right)=0 \\
H\left(x, s_{n-1} \cdots s_{0} 0\right)=f(x) .
\end{gathered}
$$

Then $H\left(x, s_{0}^{n} 0\right)=f(x)$ and $H\left(x, s_{0}^{n} 1\right)=0$. It now follows from Lemma 3.5 that there is a unique simplicial mapping $F: X \times \Delta[1] \rightarrow C^{n}(G)$ with $\hat{F}=H$. It follows (again using Lemma 3.5) that $F$ is a homotopy between $f$ and the zero mapping and Lemma 3.7 is proved.

This completes the proof of Proposition 3.4.

We conclude this section with a proof of the fact that a simplicial topological group satisfies the Kan condition.

Theorem 3.8. Let $G$ be a simplicial topological group. Then $G$ is Kan.

Proof. Let $I=\left(i_{1}, \ldots, i_{l}\right), g_{I}=\left(g_{i_{1}}, \ldots, g_{i_{l}}\right), g_{i_{j}} \in G_{q-1}$ with $\partial_{i} g_{j}=$ $\partial_{j-1} g_{i}, i<j, i, j \in I$. We need to define $\lambda_{q, I}\left(g_{I}\right) \in G_{q}$ satisfying the conditions of Definition 2.1. Let $a$ be the largest integer with $a \leq q$ and $a \notin I$. Then, either $i_{l}<a=q$ or

$$
I=\left\{i_{1}, \ldots, i_{t}, a+1, a+2, \ldots, q\right\} .
$$

(Set $t=a$ if $I=(a+1, a+2, \ldots, q)$.) Define $\beta_{1}, \ldots, \beta_{l} \in G_{q}$ inductively by

$$
\beta_{1}=s_{i_{1}} g_{i_{1}}, \quad \beta_{j}=\beta_{j-1}\left(s_{r-\varepsilon}\left(\left(\partial_{r} \beta_{j-1}\right)^{-1} g_{r}\right)\right)
$$

where $r=i_{j}, \varepsilon=0$ if $i_{j}<a$ and $r=q-j+t+1, \varepsilon=1$ for $j=$ $t+1, t+2, \ldots, l$. A straightforward computation now shows that the $\beta_{j}$ satisfy the following:

(i) $\partial_{i_{k}} \beta_{j}=g_{i_{k}}$ for $k \leq j$ if $i_{j}<a$ and for $k \leq t$ or $k \geq q-j+t+1$ if $j>t$,

(ii) if $g_{i}=\partial_{i} s_{k} g$ for $i \in I$ and some $g \in G_{g-1}$ and if $k$ or $k+1$ is in $I$, then

$$
\beta^{j}= \begin{cases}s_{k} h_{j}, & i_{j}<k, \text { for some } h_{j} \in G_{q-1}, \\ s_{k} g & \text { for } i_{j} \geq k .\end{cases}
$$

(iii) if neither $k$ nor $k+1$ is in $I$ and

$$
g_{i_{j}}= \begin{cases}s_{k-1} \bar{g}_{i_{j}} & \text { for } i_{j}<k, \\ s_{k} \bar{g}_{i_{j}-1} & \text { for } i_{j}>k+1,\end{cases}
$$

and if $\bar{\beta}_{1}, \ldots, \bar{\beta}_{l}$ are associated to $\left(\bar{g}_{i_{1}}, \ldots, \bar{g}_{i_{l}-1}\right)$, then $\beta_{j}=s_{k} \bar{\beta}_{j}$. 
It follows that $\lambda_{q, I}\left(g_{I}\right)=\beta_{l}$ satisfies the conditions of Definition 2.1. In particular, since $\beta_{l}$ is obtained from $g_{i_{1}}, \ldots, g_{i_{l}}$ using face and degeneracy operators and the group operations in $G$, it follows that $\lambda_{q, I}$ is continuous.

\section{SOME REMARKS ON TOPOLOGIES}

In this section we recall some results about compactly generated topologies and we verify that our various mappings are continuous. More explicitly, we prove Lemmas 2.7 and 2.10. We also collect some facts about Fréchet spaces which will be needed in $\S 9$.

Throughout this section all spaces are Hausdorff. If $U$ and $V$ are topological spaces, $\langle U, V\rangle$ denotes the set of continuous maps of $U$ to $V$ with the compact open topology and $U \times{ }_{c} V$ is the cartesian product of $U \times V$. We use $k(U)$ to denote $U$ with its compactly generated topology, namely, $V \subset k(U)$ is open if and only if for each compact set $C \subset U, V \cap C$ is open in $C$ with respect to the subspace topology on $C \subset U$. In the category $\mathscr{T}$ of compactly generated spaces, if $U, V \in \mathscr{T}$, one defines $U \times V=k\left(U \times{ }_{c} V\right)$ and $(U, V)=k\langle U, V\rangle$. It is proved in [21] that in the category $\mathscr{T}$, the evaluation map $U \times(U, V) \rightarrow V$ is continuous, the adjoint map $(U \times V, W) \approx(U,(V, W))$ is a homomorphism, the product $U \times V$ is the categorical product and, for topological spaces $Z$ and $W, F: Z \rightarrow W$ induces a continuous map $f: k(Z) \rightarrow k(W)$ if and only if $f \mid C$ is continuous for each compact set $C \subset Z$.

Lemma 4.1. For $X \in \mathscr{T}$, the mapping $j: X \rightarrow \Delta \mathscr{A}(X)$ is continuous.

Proof. Since $\Omega_{q}^{p}$ is metrizable, it is in $\mathscr{T}$ and $X_{q} \in \mathscr{T}$ by definition. Hence, the evaluation map gives a continuous function

$$
X_{q} \times k\left\langle X_{q}, \Omega_{q}^{p}\right\rangle \rightarrow \Omega_{q}^{p} .
$$

The map

$$
j: X_{q} \rightarrow \Delta(\mathscr{A}(X))_{q} \subset \prod\left(k\left(\mathscr{A}^{p}(X)\right), \Omega_{q}^{p}\right)
$$

has components $X_{q} \rightarrow\left(k\left(\mathscr{A}^{p}(X)\right), \Delta_{q}^{p}\right)$ whose adjoints factor as

$$
\begin{array}{ccc}
X_{q} \times k\left(\mathscr{A}^{p}(X)\right) & \rightarrow & \Omega_{q}^{p} \\
\cap & & \uparrow \\
X_{q} \times k\left(\prod\left\langle X_{r}, \Omega_{r}^{p}\right\rangle\right) & \rightarrow & X_{q} \times k\left\langle X_{r}, \Omega_{r}^{p}\right\rangle
\end{array}
$$

Lemma 4.2. If $A \in \mathscr{A} \mathscr{C}$ and $k\left(A^{p}\right)=A^{p}$ for all $p \geq 0$, then $i: A \rightarrow \mathscr{A}(\Delta(A))$ is continuous.

Proof. The mapping

$$
A^{p} \rightarrow \mathscr{A}^{p}(\Delta(A))=\left\langle\Delta(A), \Omega^{p}\right\rangle \subset \prod\left\langle\Delta(A)_{q}, \Omega_{q}^{p}\right\rangle
$$

has components with adjoint factors as follows:

$$
\begin{array}{ccc}
A^{p} \times \Delta(A)_{q} & \rightarrow & \Omega_{q}^{p} \\
\cap & & \uparrow_{e}^{p} \\
A^{p} \times \prod\left(A, \Omega_{q}\right) & \rightarrow & A^{p} \times\left(A^{p}, \Omega_{q}^{p}\right)
\end{array}
$$


By hypothesis, $k\left(A^{p}\right)=A^{p}$ so that $e$ is continuous and hence $i$ is continuous.

Let $A, B \in \mathscr{C} \mathscr{A}$ and define

$$
i_{1}: A \rightarrow A \otimes B, \quad i_{2}: B \rightarrow A \otimes B
$$

by $i_{1}(a)=a \otimes 1, i_{2}(b)=1 \otimes b$.

Lemma 4.3. For any $A, B, C \in \mathscr{A} \mathscr{C}$, the mapping

$$
i_{1}^{*} \times i_{2}^{*}:(A \otimes B, C) \rightarrow(A, C) \times(B, C)
$$

is a continuous bijection and a homeomorphism if $B^{p}$ is finite dimensional for all $p \geq 0$.

Proof. The first part of Lemma 4.3 is immediate. To prove the second part of the lemma, note that the inverse of $i_{1}^{*} \times i_{2}^{*}$ is given by

$$
\zeta:(A, C) \times(B, C) \rightarrow(A \otimes B, C),
$$

where

$$
\zeta(u, v)(a \otimes b)=u(a) v(b) .
$$

For linear spaces $U, V, W$, let $B\langle U \times V, W\rangle$ be the space of bilinear mappings from $U \times V$ to $W$ in the compact open topology. Then, on the component level, $\zeta$ factors as follows:

$$
\left\langle A^{p}, C^{p}\right\rangle \times\left\langle B^{q}, C^{q}\right\rangle \rightarrow B\left\langle A^{p} \times B^{q}, C^{p+q}\right\rangle \rightarrow\left\langle A^{p} \otimes B^{q}, C^{p+q}\right\rangle .
$$

The first of these mappings is clearly continuous and the second is easily seen to be a homeomorphism if $B^{q}$ is finite dimensional.

Lemma 4.4. For any $A, B, C$ in $\mathscr{A} \mathscr{C}$ with $B^{p}$ finite dimensional for all $p \geq 0$, the composition mapping

$$
\theta: \mathscr{F}(A, B) \times \mathscr{F}(B, C) \rightarrow \mathscr{F}(A, C)
$$

is continuous. Furthermore the composite

$$
\mathscr{F}(A, \mathscr{A}(X)) \times X \stackrel{\text { id } \times j}{\longrightarrow} \mathscr{F}(A, \mathscr{A}(X)) \times \mathscr{F}(\mathscr{A}(X), R) \stackrel{\theta}{\rightarrow} \mathscr{F}(A, R)
$$

is continuous.

Proof. Consider the commutative diagram

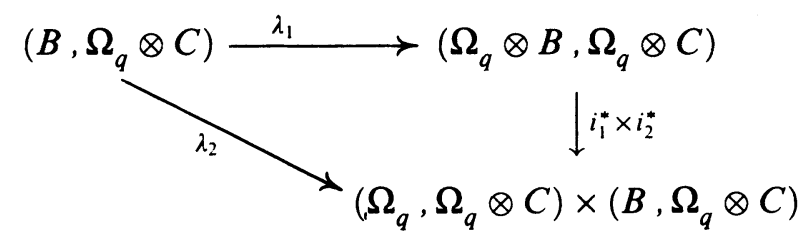

where

$$
\lambda_{1}(u)(\omega \otimes b)=(\omega \otimes 1) u(b), \quad \lambda_{2}(u)=(\mathrm{id} \otimes 1, u) .
$$


Now, $\lambda_{2}$ is clearly continuous so using Lemma 4.3 , we have $\lambda_{1}$ continuous. Thus the composite

$$
\begin{gathered}
\mathscr{F}(A, B)_{q} \times \mathscr{F}(B, C)_{q}=\left(A, \Omega_{q} \otimes B\right) \times\left(B, \Omega_{q} \otimes C\right) \\
\downarrow 1 \times \lambda_{1} \\
\left.\left(A, \Omega_{q} \otimes B\right) \times\left(\Omega_{q} \otimes B\right), \Omega_{q} \otimes C\right) \\
\downarrow \\
\mathscr{F}(A, C)_{q}=\left(A, \Omega_{q} \otimes C\right)
\end{gathered}
$$

is continuous.

To prove the second assertion, we must verify that

$$
X_{q} \rightarrow\left(\mathscr{A}^{r}(X), \Omega_{q}^{r}\right) \rightarrow\left(\Omega_{q}^{r} \times \mathscr{A}^{p}(X), \Delta_{q}^{r+p}\right)
$$

is continuous when $B=\mathscr{A}(X)$ and $C=R$. Under this map, the image of $x \in X_{q}$ under $w \times u \in \Delta_{q}^{r} \times \mathscr{A}^{p}(X)$ is $w u(x)$, that is, multiplication and evaluation. This is continuous because evaluation is continuous on compact spaces.

We now state some facts about Fréchet spaces which will be needed in $\S 9$.

Recall that a Fréchet space is a metrizable topological vector space which is complete and locally convex. In particular, any Banach space is a Fréchet space. All Fréchet spaces have the following properties:

(4.5) Any closed subspace of a Fréchet space is a Fréchet space.

(4.6) Any quotient of a Fréchet space by a closed subspace is a Fréchet space.

These facts are elementary. (See [23, p. 85].)

(4.7) If $X$ is locally compact and countable at infinity and $V$ is a Fréchet space, then $(X, V)$, the space of continuous functions from $X$ to $V$, in the compact-open topology, is a Fréchet space.

If $K_{1} \subset K_{2} \subset \cdots \subset K_{n} \subset \cdots$ is a sequence of compact sets with union $X$, then

$$
\|f\|_{n}=\sup _{x \in K_{n}}\|f(x)\|
$$

defines a sequence of seminorms on $(X, V)$ which can be used to define a metric. Verifying completeness and local convexity is not difficult.

(4.8) If $V_{1}$ and $V_{2}$ are Fréchet spaces and $\alpha: V_{1} \rightarrow V_{2}$ is a continuous epimorphism, then there is a continuous (not necessarily linear) $\sigma: V_{2} \rightarrow V_{1}$ with $\alpha \sigma=$ identity.

This result is stated without proof in [2, p. 258] and proved for Banach spaces in [1]; see also [14]. The proof given in [14] can be modified in a straightforward way to prove (4.8) using the fact that any Fréchet space has a neighborhood system $U_{1} \supset U_{2} \supset \cdots$ of 0 with each $U_{i}$ convex and with $U_{i}+U_{i} \subset U_{i+1}$.

\section{Proof of the de Rham theorem}

The proofs of most of the lemmas in this section are trivial and omitted or given very briefly. We begin with some preliminaries about the simplicial differential graded algebra $\Omega$. (Compare Dupont [25].) 
Let $\Omega\left(\Delta^{q} \times I\right)$ be the $C^{\infty}$ differential forms on $\Delta^{q} \times I$. If $t$ denotes the coordinate function on $I$, then $\omega \in \Omega\left(\Delta^{q} \times I\right)^{p}$ can be written as $\omega=\omega_{1}(t)+$ $\omega_{2}(t) d t$, where $\omega_{1}(t) \in \Omega_{q}^{p}$ and $\omega_{2}(t) \in \Omega_{q}^{p-1}$. Define

$$
\mu: \Omega\left(\Delta^{q} \times I\right)^{p} \rightarrow \Omega_{q}^{p-1}
$$

to be the usual "integration along fibres" mapping,

$$
\mu(\omega)=(-1)^{p-1} \int_{0}^{1} \omega_{2}(t) d t .
$$

A straightforward computation proves the following.

Lemma 5.1. Let $i_{0}, i_{1}: \Delta^{q} \rightarrow \Delta^{q} \times I$ be given by

$$
i_{0}(x)=(x, 0), \quad i_{1}(x)=(x, 1) .
$$

Then

$$
d \mu+\mu d=i_{1}^{*}-i_{0}^{*}, \quad \partial_{i} \mu=\mu\left(e_{i} \times \mathrm{id}\right)^{*}, \quad s_{i} \mu=\mu\left(d_{i} \times \mathrm{id}\right)^{*} .
$$

Here, $d$ is the exterior differential, $\partial_{i}, s_{i}$ are the face and degeneracy mappings, and $e_{i}: \Delta^{q-1} \rightarrow \Delta^{q}, d_{i}: \Delta^{q+1} \rightarrow \Delta^{q}$ are the usual face inclusions and degeneracy projections defined in $\S 2$.

For the remainder of this section only, we define $\Omega_{q}^{-1}=R$ with $\partial_{i}=s_{i}=$ id and $d: \Omega_{q}^{-1} \rightarrow \Omega_{q}^{0}$ by $d(r)=r$, the constant function. Let $b_{i}: \Delta^{q} \times \Delta^{1} \rightarrow \Delta^{q}$ be given by

$$
b_{i}(x, t)=t x+(1-t) v_{i},
$$

where $v_{i}$ is the $i$ th vertex of $\Delta^{q}$ and let $\mu_{i}: \Omega_{q}^{p} \rightarrow \Omega_{q}^{p-1}$ be given by

$$
\mu_{i}(\omega)= \begin{cases}\mu b_{i}^{*} \omega, & p>0, \\ \omega\left(v_{i}\right), & p=0 .\end{cases}
$$

Lemma 5.2. The functions $\mu_{i}$ satisfy the following.

$$
\begin{gathered}
d \mu_{i}+\mu_{i} d=\mathrm{id}, \\
\partial_{j} \mu_{i}= \begin{cases}\mu_{i} \partial_{j}, & i<j, \\
\mu_{i-1} \partial_{j}, & i>j,\end{cases} \\
\mu_{i} s_{j}= \begin{cases}s_{j} \mu_{i}, & i \leq j, \\
s_{j} \mu_{i-1}, & i>j .\end{cases}
\end{gathered}
$$

The proof is trivial.

For $I=\left(i_{0}, \ldots, i_{p}\right)$, let $\mu_{I}$ be defined by

$$
\mu_{I}=\mu_{i_{p}} \mu_{i_{p-1}} \cdots \mu_{i_{0}} .
$$

An easy induction proves the following. 
Lemma 5.3. For $I=\left(i_{0}, \ldots, i_{p}\right) \in \Delta[q]_{p}$, we have

$$
d \mu_{I}+(-1)^{p} \mu_{I} d=(-1)^{p} \sum_{j=0}^{p}(-1)^{j} \mu_{\partial_{j} I}
$$

where $\partial_{j}$ denotes the jth face operator in $\Delta[q]$.

If $I=\left(i_{0}, i_{1}, \ldots, i_{p}\right) \in \Delta[p]_{q}$, let $\beta_{I} \in \Omega_{q}^{q}$ be defined by

$$
\beta_{I}=\sum_{j=0}^{p}(-1)^{j} t_{i_{j}} d t_{i_{0}} \cdots \widehat{d} t_{i_{j}} \cdots d t_{i_{p}}
$$

where $t_{0}, \ldots, t_{q}$ are the barycentric coordinates in $\Delta^{q}$.

Lemma 5.4. For $I \in \Delta[q]_{p}$ and $0 \leq j \leq p$, we have

$$
\partial_{i} \beta_{I}=\sum_{e_{i} J=I} \beta_{J}, \quad s_{i} \beta_{I}=\sum_{d_{i} J=I} \beta_{J} .
$$

The proof is straightforward.

Define mappings

$$
\psi: \Omega_{q}^{p} \rightarrow C_{q}^{p}(R), \quad \varphi: C_{q}^{p}(R) \rightarrow \Omega_{q}^{p}, \quad \gamma: \Omega_{q}^{p} \rightarrow \Omega_{q}^{p-1},
$$

by

$$
\psi(\omega)(I)=\mu_{I}(\omega), \quad \varphi(u)=\sum_{I \in \Delta[q]_{p}} p ! u(I) \beta_{I}, \quad \gamma(\omega)=\sum_{p \leq q} p ! \sum_{I \in \Delta[q]_{p}} \mu_{I}(\omega) \beta_{I} .
$$

Lemma 5.5. The mappings $\psi, \varphi$, and $\gamma$ define simplicial mappings which satisfy the following.

$$
\begin{aligned}
\psi d=\delta \psi, \quad \varphi \delta & =d \varphi, \\
\psi \varphi=\mathrm{id}, \quad d \gamma+\gamma d & =\varphi \psi-\mathrm{id} .
\end{aligned}
$$

Proof. The fact that $\psi$ is simplicial follows immediately from Lemma 5.2. To see that $\varphi$ and $\gamma$ are simplicial, one uses Lemma 5.2 and 5.4. The equation $\psi d=\delta \psi$ is an easy consequence of Lemma 5.4.

To prove that $\psi \varphi=\mathrm{id}$, we first note that in terms of coordinates, $b_{j}: \Delta_{q} \times$ $I \rightarrow \Delta_{q}$ is given by

$$
b_{j}\left(t_{0}, \ldots, t_{q}, t\right)_{k}=t t_{k}+\delta_{j k}(1-t),
$$

where $\delta_{j k}=1$ if $j=k$ and zero otherwise. Hence

$$
\begin{aligned}
b_{j}^{*} \beta_{I}= & t^{p+1} \beta_{I}+t^{p}(1-t) d t_{i_{0}} \cdots d \hat{t}_{i_{j}} \cdots d t_{i_{p}} \\
& +\left(\sum \delta_{j i_{k}}(-1)^{p+k+1} t^{p-1} \beta_{\partial_{k} I}\right) d t
\end{aligned}
$$

and

$$
\mu_{j} \beta_{I}= \begin{cases}\frac{(-1)^{k}}{p} \beta_{\partial_{k} I} & \text { if for some } k, j=i_{k} \\ 0 & \text { if } j \notin I .\end{cases}
$$


Thus $\mu_{J} \beta_{I}=1 / p$ ! if $J=I$ and is zero if $J \neq I$. Therefore, $\psi \varphi=\mathrm{id}$.

We next show that $\varphi \delta=d \varphi$. For any tuple $J$ of integers between 0 and $q, J=\left(j_{0}, j_{1}, \ldots, j_{p}\right)$, let $\beta_{J}=(\operatorname{sign} \alpha) \beta_{I}$, where $I=\left(j_{\alpha(0)}, \ldots, j_{\alpha(p)}\right)$, $j_{\alpha(0)} \leq j_{\alpha(1)} \leq \cdots \leq j_{\alpha(0)}$. Note that the ambiguity of $\alpha$ does not matter because $\beta_{I}=0$ if the entries of $I$ are not distinct. Then

$$
\begin{aligned}
\sum_{k \notin I} \beta_{[k, I]} & =\sum_{k} \beta_{[k, I]}=\sum t_{k} d t_{i_{0}} \cdots d t_{i_{p}}+d t_{k} \beta_{I} \\
& =\left(\sum t_{k}\right) d t_{i_{0}} \cdots d t_{i_{p}}+\left(\sum d t_{k}\right) \beta_{I} \\
& =d t_{i_{0}} \cdots d t_{i_{p}}=\frac{1}{p+1} d \beta_{I}
\end{aligned}
$$

and

$$
\begin{aligned}
d \varphi u & =d \sum p ! u(I) \beta_{I}=(p+1) \sum_{I, k \notin I} u(I) \beta_{[k, I]} \\
& =\sum u\left(\partial_{i} J\right)(-1)^{i}(p+1) ! \beta_{J}=\varphi \delta u .
\end{aligned}
$$

Using Lemma 5.3 and the above argument one can show that $d \nu+\nu d=\varphi \psi-\mathrm{id}$.

For $X \in \Delta \mathscr{T}$, composition with $\psi, \varphi$, and $\gamma$ gives mappings

$$
\psi: \mathscr{A}(X) \rightarrow C(X, R), \quad \varphi: C(X ; R) \rightarrow \mathscr{A}(X), \quad \gamma: \mathscr{A}(X) \rightarrow \mathscr{A}(X)
$$

satisfying the identities of Lemma 5.5. Thus we have

Theorem 5.6. The mapping $\psi: \mathscr{A}(X) \rightarrow C(X, R)$ defines a vector space isomorphism

$$
\psi_{*}: H_{d R}^{*}(X) \rightarrow H^{*}(X ; R) .
$$

The point of the above exposition is to develop the functions $\psi, \varphi$, and $\gamma$ (which we will use in the next section) rather than to prove Theorem 5.6. We now show that $\psi_{*}$ is an algebra isomorphism. Let $C^{r, s} \in \Delta \mathscr{T}$ be defined by

$$
C_{q}^{r, s}= \begin{cases}C^{r}\left(\Delta[q] ; \Omega_{q}^{s}\right), & s \geq-1, r \geq 0, \\ \Omega_{q}^{s}, & r=-1, s \geq 0, \\ 0, & \text { otherwise }\end{cases}
$$

and

$$
d_{1}: C_{q}^{r, s} \rightarrow C_{q}^{r+1, s}, \quad d_{2}: C_{q}^{r, s} \rightarrow C_{q}^{r, s+1}
$$

by

$$
d_{1} u(I)=\sum_{j=0}^{r}(-1)^{j} u\left(\partial_{j} I\right), \quad d_{2} u(I)=(-1)^{r} d(u(I)) .
$$

Define mappings

$$
\begin{gathered}
\gamma_{1}: C_{q}^{r, s} \rightarrow C_{q}^{r-1, s}, \quad \gamma_{2}: C_{q}^{r, s} \rightarrow C_{q}^{r, s-1}, \\
\psi: C_{q}^{r, s} \rightarrow C_{q}^{r+s}(R), \quad \rho: C_{q}^{r, s} \otimes C_{q}^{l, m} \rightarrow C_{q}^{r+l, s+m}
\end{gathered}
$$


by

$$
\begin{aligned}
\gamma_{1}(u)(I) & =\sum_{j=0}^{q} t_{j} u(j, I), \\
\gamma_{2}(u)(I) & =\mu_{i_{0}} u\left(i_{1}, \ldots, i_{r}\right), \\
\psi(u)(I) & =\mu_{\left(i_{0}, \ldots, i_{q}\right)} u\left(i_{r}, \ldots, i_{r+s}\right), \\
\rho(u \otimes v)(I) & =u\left(i_{0}, \ldots, i_{r}\right) v\left(i_{r}, \ldots, i_{r+l}\right) .
\end{aligned}
$$

Lemma 5.7. The mappings $d_{1}, d_{2}, \gamma_{1}, \gamma_{2}, \psi$, and $\rho$ defined above are simplicial and satisfy the identities

$$
\begin{gathered}
d_{1}^{2}=d_{2}^{2}=d_{1} d_{2}+d_{2} d_{1}=0, \\
d_{1} \gamma_{1}+\gamma_{1} d_{1}=\mathrm{id}, \quad d_{2} \gamma_{2}+\gamma_{2} d_{2}=\mathrm{id}, \\
\psi\left(d_{1}+d_{2}\right)=\delta \psi .
\end{gathered}
$$

Furthermore, $d_{1}+d_{2}$ is a derivation in the graded sense with respect to the multiplication $\rho$.

The proof is tedious but straightforward.

It follows from Lemma 5.7 that $\left\{C^{r, s} ; d_{1}, d_{2}\right\}$ is a simplicial double complex. Note that $C(R)_{q}^{p}=C_{q}^{p,-1}$ and $\Delta_{q}^{p}=C_{q}^{-1, p}$. Let $\bar{C}_{q}^{n}$ and $\bar{d}: \bar{C}_{q}^{n} \rightarrow \bar{C}_{q}^{n+1}$ be the simplicial chain complex defined by

$$
\bar{C}_{q}^{n}=\sum_{0 \leq r \leq n} C_{q}^{r, n-r}, \quad \bar{d}=d_{1}+d_{2}
$$

and define mappings $\alpha: \Delta \rightarrow \bar{C}, \quad \beta: C(R) \rightarrow \bar{C}$ by

$$
\alpha=d_{1}: C^{-1, p} \rightarrow C^{0, p}, \quad \beta=d_{2}: C^{p_{1}-1} \rightarrow C^{p, 0} .
$$

For $X \in \mathscr{T}$, let $\bar{C}(X)=(X, \bar{C}) \in \mathscr{A}$. Composition with the mappings in Lemma 5.7 gives mappings on $\bar{C}(X)$ satisfying the same identities.

Proposition 5.8. In the diagram

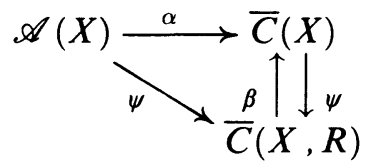

one has $\zeta \alpha=\psi, \psi \beta=$ identity, and $\alpha$ and $\beta$ are ring homomorphisms inducing isomorphisms on $H_{*}$.

Proof. Using the chain homotopies $\gamma_{1}$ and $\gamma_{2}$, we see that, for any $p \geq 0$, the sequences

$$
\begin{aligned}
& 0 \rightarrow \mathscr{A}^{p}(X) \stackrel{\alpha}{\rightarrow} C^{0, p} \stackrel{d_{1}}{\rightarrow} C^{1, p} \rightarrow \cdots, \\
& 0 \rightarrow C^{p}(X, R) \stackrel{\beta}{\rightarrow} C^{p, 0} \stackrel{d_{2}}{\rightarrow} C^{p, 1} \rightarrow
\end{aligned}
$$

are exact. Standard results about double complexes now show that

$$
\alpha_{*}: H_{*}(\mathscr{A}(X)) \rightarrow H^{*}(\bar{C}), \quad \beta_{*}: H_{*}\left(C(X ; R) \rightarrow H_{*}(\bar{C})\right.
$$

are isomorphisms. 
Corollary 5.9. The mapping $\psi: \mathscr{A}(X) \rightarrow C(X ; R)$ induces an algebra isomorphism

$$
\psi_{*}: H_{d R}^{*}(X) \rightarrow H^{*}(X ; R)
$$

\section{FIBRE SPACES}

We now introduce the notion of fibration in $\Delta \mathscr{T}$ and develop some of its properties. We also define twisted cartesian products and prove some results about twisted cartesian products with fibres $K(R, n)$ (and its differential form analogue) which will be useful in what follows.

Let $p: E \rightarrow B$ be a map in $\Delta \mathscr{T}$ and let $I=\left\{i_{1}, \ldots, i_{2}, \ldots, i_{l}\right\}$, where $0 \leq$ $i_{1}<i_{2}<\cdots<i_{l} \leq q$. Define $E(q, I)$ to be the subspace of $B_{q} \times E_{q-1}^{l}\left(E_{q-1}^{l}\right.$ is the $l$-fold product of $\left.E_{q-1}\right)$ consisting of all $\left(b, y_{i_{1}}, \ldots, y_{i_{l}}\right)$ satisfying

$$
\partial_{i} b=p y_{i}, \quad \partial_{i} y_{j}=\partial_{j-1} y_{i}
$$

for $i, j \in I, i<j$. Let $p_{q, I}: E_{q} \rightarrow E(q, I)$ be given by

$$
p_{q, I}(y)=\left(p y, \partial_{i_{1}} y, \ldots, \partial_{i_{l}} y\right) \text {. }
$$

If $k \notin I$, define $\bar{s}_{k} I=\left\{j_{1}, \ldots, j_{l}\right\}$ where

$$
j_{m}= \begin{cases}i_{m} & \text { for } i_{m}<k \\ i_{m}+1 & \text { for } i_{m}>k\end{cases}
$$

Definition 6.1. A map $p: E \rightarrow B$ in $\Delta \mathscr{T}$ is a fibration if, for each $q \geq 0$ and $I=\left\{i_{1}, \ldots, i_{l}\right\}$ as above, $1 \leq l \leq q$, there are mappings

$$
\lambda_{q, I}: E(q, I) \rightarrow E_{q}
$$

satisfying

(i) $\lambda_{q, I}$ is continuous,

(ii) $p_{q, I} \lambda_{q, I}=$ identity,

(iii) if $k$ or $k+1 \in I$, then, for all $y \in E_{q-1}$,

$$
\lambda_{q, I} p_{q, I}\left(s_{k} y\right)=s_{k} y \text {, }
$$

(iv) if $k, k+1 \notin I$ and hence $I=s_{k} I^{\prime}$, then for all $y \in E_{q-1}$,

$$
\lambda_{q, I} p_{q, I}\left(s_{k} y\right)=s_{k} \lambda_{q-1, I^{\prime}} p_{q-1, I^{\prime}}(y) .
$$

Remark. The definition of a fibration in the category of simplicial sets involves only condition (ii) for $I$ of the form $\{0,1, \ldots, j-1, j+1, \ldots, q\}$. It is easy to see [13, Lemma 6.8] that this implies condition (ii) in the category $\Delta \mathscr{S}$ for all $I$. This implication does not hold in the category $\Delta \mathscr{G}$ because of the continuity requirement. Conditions (iii) and (iv) assert that $\lambda_{q, I}\left(b, y_{I}\right)$ is degenerate whenever $\left(b, y_{I}\right)$ is such as to make this possible, in which case $\lambda_{q, I}$ commutes with the degeneracy operations.

Of course, $X \in \Delta \mathscr{T}$ is Kan if and only if the mapping $X \rightarrow \mathrm{pt}$ is a fibration. 
Theorem 6.2. If $p: E \rightarrow B$ is a fibration in $\Delta \mathscr{T}$, and $X \in \mathscr{T}$, then $\mathscr{F}(X, E)$ $\rightarrow \mathscr{F}(X, B)$ is a fibration in $\Delta \mathscr{T}$.

Corollary 6.3. If $X, Y \in \Delta \mathscr{T}$ and $Y$ is Kan, then $\mathscr{F}(X, Y)$ is Kan.

Corollary 6.4. If $X, Y \in \Delta \mathscr{T}$ and $Y$ is Kan, then homotopy is an equivalence relation.

The proof of Theorem 6.2 is given in $\S 12$.

The usual arguments give

Theorem 6.5. If $p: E \rightarrow B$ is a fibration and $B$ is Kan, then $E$ is Kan and for any $b_{0} \in B, F=p^{-1}\left(b_{0}\right)$ is $K$ an and there is an exact sequence

$$
\cdots \rightarrow \pi_{n}\left(F, y_{0}\right) \stackrel{i_{*}}{\rightarrow} \pi_{n}\left(E, y_{0}\right) \stackrel{j_{*}}{\rightarrow} \pi_{n}\left(B, b_{0}\right) \stackrel{\partial_{*}}{\rightarrow} \pi_{n-1}\left(F, y_{0}\right) \rightarrow \cdots,
$$

where $y_{0} \in F$ and $i_{*}, j_{*}$ and $\partial_{*}$ are continuous homomorphisms.

We will also need the following variant on this result in the next section.

Proposition 6.6. Let $p^{\prime}: Y^{\prime} \rightarrow X^{\prime}$ be a fibration with $X^{\prime}$ connected and $Y^{\prime}$ contractible. Let $\hat{f}: \tilde{X} \rightarrow X^{\prime}$ be a mapping and $\tilde{p}: \tilde{Y} \rightarrow \tilde{X}$ the induced fibration. Then, for any component $X$ of $\tilde{X}$, we have an exact sequence

$$
\cdots \rightarrow \pi_{q}\left(Y, y_{0}\right) \stackrel{p_{*}}{\rightarrow} \pi_{q}\left(X, x_{0}\right) \stackrel{f_{*}}{\rightarrow} \pi_{q}\left(X^{\prime}, x_{0}^{\prime}\right) \rightarrow \cdots \rightarrow \pi_{1}\left(X^{\prime}, x_{0}^{\prime}\right) \stackrel{\partial_{*}}{\rightarrow} \pi_{0} Y \rightarrow *,
$$

where $Y=\tilde{p}^{-1} X, p=\tilde{p}|Y, f=\tilde{f}| X$.

Proof. If we replace $f: X \rightarrow X^{\prime}$ by a fibration, one sees easily that the fibre of this fibration has the homotopy type of $Y$ (since $Y^{\prime}$ is contractible). The sequence above is the exact homotopy sequence of this fibration.

Note that $\pi_{1}\left(X^{\prime}, x_{0}^{\prime}\right)$ acts on $\pi_{0} Y$ and exactness at $\pi_{1}\left(X^{\prime}, x_{0}^{\prime}\right)$ asserts that $\partial_{*} a=\partial_{*} b$ if and only if there is an $\alpha \in \pi_{1}\left(X^{\prime}, x_{0}^{\prime}\right)$ with $\alpha \cdot a=b$.

We next introduce a particular class of fibre spaces, the twisted cartesian products (TCP).

Let $B$ and $F$ be simplicial spaces and $G$ a simplicial group acting continuously on the left of $F$. A twisting function $\tau$ is a sequence

$$
\tau=\tau_{q}: B_{q} \rightarrow G_{q-1}
$$

of continuous mappings, $q>0$, satisfying the usual identities:

$$
\begin{aligned}
& \tau\left(\partial_{1} b\right)=\tau\left(\partial_{0} b\right) \partial_{0} \tau(b), \\
& \tau\left(\partial_{i} b\right)=\partial_{i-1} \tau(b), \quad i>1 \text {, } \\
& \tau\left(s_{i} b\right)= \begin{cases}e_{q-1}, & i=0, \\
s_{i-1} \tau(b), & i>0 .\end{cases}
\end{aligned}
$$

Here $e_{q-1} \in G_{q-1}$ is the identity. The twisted cartesian product (TCP) $B \times_{\tau} F$ is the simplicial space with

$$
\left(B \times{ }_{\tau} F\right)_{q}=B_{q} \times F_{q}
$$


and whose face and degeneracy mappings are the products of those in $B$ with those in $F$ except that

$$
\partial_{0}(b, y)=\left(\partial_{0} b, \tau(b) \partial_{0} y\right)
$$

(See [13] for example.)

Lemma 6.7. If $F$ is Kan, then $\pi: B \times{ }_{\tau} F \rightarrow B, \pi(b, y)=b$, is a fibration.

Proof. Let $E=B \times{ }_{\tau} F$ and $\left(b, z_{i_{1}}, \ldots, z_{i_{1}}\right) \in E(q, I)$. If $z_{i}=\left(b_{i}, y_{i}\right)$ for $i \in I$, then $b_{i}=\partial_{i} b$ and

$$
\partial_{i} y_{j}= \begin{cases}\partial_{j-1} y_{i}, & 0<i<j \\ \tau\left(b_{j}\right)^{-1} \partial_{0} y_{j}, & i=0, j>1, \\ \partial_{0} \tau(b)^{-1} \partial_{0} y_{1}, & i=0, j=1 .\end{cases}
$$

If $x_{i} \in F_{q-1}$ for $i \in I$ are defined by

$$
x_{j}= \begin{cases}s_{0} \tau\left(b_{j}\right) y_{j}, & j>1, \\ \tau(b) y_{1}, & j=1, \\ y_{0}, & j=0,\end{cases}
$$

then $\partial_{i} x_{j}=\partial_{j-1} x_{i}$ for $i, j \in I, i<j$. Let

$$
\tilde{\lambda}=\tilde{\lambda}_{q, I}: F(q, I) \rightarrow F_{q}
$$

be the mapping which exists since $F$ is Kan and define

$$
\lambda=\lambda_{q, I}: E(q, I) \rightarrow E_{q}
$$

by

$$
\lambda\left(b, z_{i}\right)=\left(b, s_{0} \tau(b)^{-1} \tilde{\lambda}\left(x_{I}\right)\right) .
$$

This $\lambda$ is easily seen to satisfy conditions (i)-(iv) of Definition 6.1.

The following two lemmas define basic fibrations which are analogues of the path space fibration over Eilenberg-Mac Lane spaces in the category $\mathscr{T}$.

For $\Gamma \in \Delta \mathscr{A}$ let $C_{n}(\Gamma), Z_{n}(\Gamma)$ and $B_{n}(\Gamma) \in \Delta \mathscr{T}$ be defined by

$$
\begin{aligned}
& C_{n}(\Gamma)_{q}=\Gamma_{q}^{n}, \\
& Z_{n}(\Gamma)_{q}=\left\{u \in \Gamma_{q}^{n} \mid d u=0\right\}, \\
& B_{n}(\Gamma)_{q}=\left\{d v \mid v \in \Gamma_{q}^{n-1}\right\} .
\end{aligned}
$$

Lemma 6.8. If $A \in \mathscr{A} \mathscr{C}$, then

$$
d: C_{n}(\Omega \otimes A) \rightarrow Z_{n+1}(\Omega \otimes A)
$$

is a twisted cartesian product with group and fibre $Z_{n}(\Omega \otimes A)$.

Proof. For $q \geq 0$, consider the exact sequence

$$
0 \rightarrow Z_{n}\left(\Omega_{q} \otimes A\right) \stackrel{j}{\rightarrow} C_{n}\left(\Omega_{q} \otimes A\right) \rightarrow Z_{n+1}\left(\Omega_{q} \otimes A\right) \rightarrow 0 .
$$


According to Lemma 5.2, the homomorphism

defines a splitting

$$
\mu_{0}: \Omega_{q}^{n} \rightarrow \Delta_{q}^{n-1}
$$

$$
\tilde{\mu}_{0}=\mu_{0} \otimes \mathrm{id}: Z_{n+1}\left(\Omega_{q} \otimes A\right) \rightarrow C_{n}\left(\Omega_{q} \otimes A\right)
$$

of this sequence. Furthermore, $\tilde{\mu}_{0} s_{i}=s_{i} \tilde{\mu}_{0}$ for all $i$ and $\tilde{\mu}_{0} \partial_{i}=\partial_{i} \tilde{\mu}_{0}$ for $i>0$. Let

$$
\tau: Z_{n+1}\left(\Omega_{q} \otimes A\right) \rightarrow Z_{n}\left(\Omega_{q-1} \otimes A\right)
$$

be defined by

$$
\tau(\alpha)=\partial_{0} \tilde{\mu}_{0}(\alpha)-\tilde{\mu}_{0}\left(\partial_{0} \alpha\right) .
$$

Then $\tau$ is a twisting function and the mapping

$$
K: Z_{n+1}\left(\Omega_{q} \otimes A\right) \times{ }_{\tau} Z_{n}\left(\Omega_{q} \otimes A\right) \rightarrow C_{n}\left(\Omega_{q} \otimes A\right)
$$

given by $K(\alpha, \beta)=\tilde{\mu}_{0}(\alpha)+\beta$ is an isomorphism with $d K=\pi$ where $\pi: Z_{n+1} \times$ $Z_{n} \rightarrow Z_{n+1}$ is projection onto the first factor.

The same argument can be applied to $C(R)$ using the mapping

$$
\mu_{0}: C_{q}^{n+1}(R) \rightarrow C_{q}^{n}(R)
$$

given by $\mu_{0}(u)\left(i_{0}, \ldots, i_{n}\right)=u\left(0, i_{0}, \ldots, i_{n}\right)$. We then have

Lemma 6.9. The mapping

$$
\delta: C^{n+1}(R) \rightarrow Z_{n+1}(C(R))
$$

is a twisted cartesian product with group and fibre $Z_{n}(C(R))$.

Lemma 6.10. For any $n$ and $A \in \mathscr{A} \mathscr{C}, C_{n}(\Omega \otimes A)$ and $C^{n}(R)$ are contractible. Proof. Let $F: C_{n}(\Omega \otimes A) \times \Delta[1] \rightarrow C(\Omega \otimes A)$ be defined as follows: If $w \in$ $C_{n}(\Omega \otimes A)_{q}$, and $s \in \Delta[1]_{q}$, then, viewing $s$ as a mapping $s: \Delta[q] \rightarrow \Delta[1]$ let $F(w, s)=\left(s^{*} t_{1}\right) w$, where $\left(s^{*} t_{1}\right)(u \otimes a)=\left(\left(s^{*} t\right) u\right) \otimes a$. Then $F$ is the desired contraction. The proof that $C^{n}(R)$ is contractible proceeds in the same way.

Recall that if $A \in \mathscr{A} \mathscr{C}$ and $a \in A^{n+1}$ with $d a=0$, we can form $A[x]=$ $A_{a}[x]$, where $x$ has degree $n$ and $d x=a$. In this situation, we have a commutative diagram of algebras

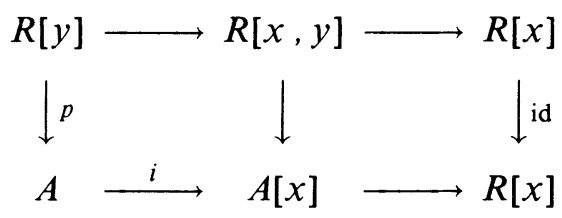

where $p(y)=a$ and $d y=d x=0$ in $R[y]$ and $R[x]$ while $d x=y$ in $R[x, y]$. For any $B \in \mathscr{A} \mathscr{C}$, we apply the functor $\mathscr{F}(, B)$ to this diagram in which case the top row becomes the TCP

$$
Z_{n}(\Omega \otimes B) \rightarrow C_{n}(\Omega \otimes B) \stackrel{d}{\rightarrow} Z_{n+1}(\Omega \otimes B)
$$

of Lemma 6.8. 
Lemma 6.12. The mapping

$$
\mathscr{F}(i, B): \mathscr{F}(A[x], B) \rightarrow \mathscr{F}(A, B)
$$

is the TCP with fibre $Z_{n}(\Omega \otimes B)$ induced from (6.11) by the mapping

$$
\mathscr{F}(p, B): \mathscr{F}(A, B) \rightarrow \mathscr{F}(R[y], B)=Z_{n+1}(\Omega \otimes B) .
$$

Proof. The statement of this lemma is clearly true in the category of simplicial sets. We need only show that the mapping

$$
\mathscr{F}(A[x], B)_{q} \rightarrow\left\{(u, v) \in\left(A, \Omega_{q} \otimes B\right) \times C_{n}\left(\Omega_{q} \otimes B\right): v=u p\right\}
$$

is a homeomorphism. Since $A[x]=A \otimes R[x]$ as algebras, this follows from Lemma 4.3.

Corollary 6.13. If $A$ is $F N F$, then

$$
\mathscr{F}(A, B) \rightarrow \mathscr{F}(R, B)=\text { point }
$$

is a fibration for any $B$. Thus $\mathscr{F}(A, B)$ and $\Delta(A)=\mathscr{F}(A, R)$ are Kan.

\section{Proof of Theorem 2.20}

The proof of each of the statements in Theorem 2.20 follows the same course. We carry out in detail the proof that

$$
\Delta: \mathscr{F}(A, B) \rightarrow \mathscr{F}(\Delta B, \Delta A)
$$

is a weak equivalence, where $A, B \in \mathscr{A} \mathscr{C}$ are FNF, dealing with the remaining two statements only when differences in the proofs require us to do so.

The idea of the proof is to show, by a sequence of reductions, that the theorem is true for general $A$ if it is true for $A=R[y]$.

Remark. Each of the three statements of Theorem 2.20 involve proving that a continuous simplicial mapping induces isomorphisms on homotopy groups. Since the homotopy groups $\pi_{q}(X)$ of a simplicial space $X$ are defined to be the homotopy groups of the underlying simplicial set $X^{\delta}$ (with topology on $\pi_{q}(X)$ induced from the topology on $X$ ), we can ignore the topology on the simplicial spaces that occur and work in the category of simplicial sets. We will do so for the remainder of the section without further mention.

Suppose now that $A$ and $B$ are FNF and consider the diagram

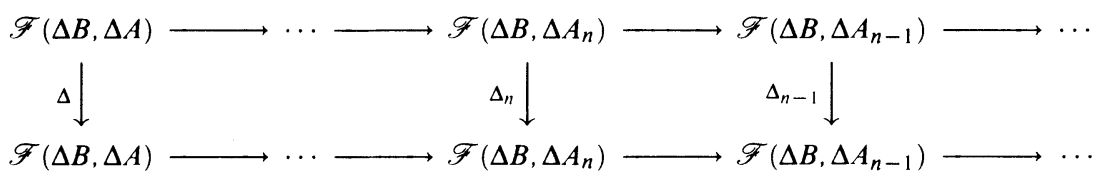

where $A=\lim A_{n}, A_{n}=A_{n-1}\left[x_{n}\right]$ as in Definition 2.5. According to Lemma 6.11 , each of the mappings

$$
\pi: \mathscr{F}\left(A_{n}, B\right) \rightarrow \mathscr{F}\left(A_{n-1}, B\right)
$$


is a fibration with fibre $\mathscr{F}(R[x], B), \operatorname{deg} x=\operatorname{deg} x_{n}$. Setting $B=R$ in this same lemma, we see that $\Delta A_{n} \rightarrow \Delta A_{n-1}$ is a fibration with fibre $\Delta R[x]$. Therefore, using Theorem 6.2 we see that

$$
\pi^{\prime}: \mathscr{F}\left(\Delta B, \Delta A_{n}\right) \rightarrow \mathscr{F}\left(\Delta B, \Delta A_{n-1}\right)
$$

is a fibration with fibre $\mathscr{F}(\Delta B, \Delta R[x])$. Thus, according to [4, Theorem 3.1, p. 254], we have a commutative diagram

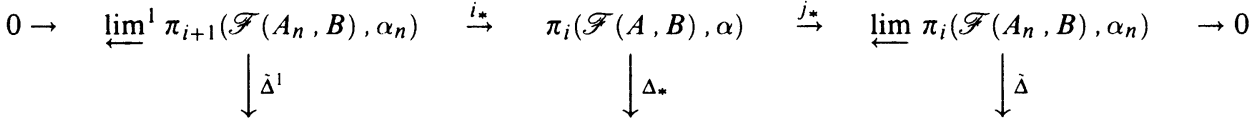

$0 \rightarrow \lim ^{1} \pi_{i+1}\left(\mathscr{F}\left(\Delta B, \Delta A_{n}\right), \alpha_{n}^{\prime}\right) \stackrel{i_{*}^{\prime}}{\rightarrow} \pi_{i}\left(\mathscr{F}(\Delta B, \Delta A), \alpha^{\prime}\right) \stackrel{j_{*}^{\prime}}{\rightarrow} \lim \pi_{i}\left(\mathscr{F}\left(\Delta B, \Delta A_{n}\right), \alpha_{n}^{\prime}\right) \rightarrow 0$

for each $i>0$, where each of the horizontal sequences is exact and $\tilde{\Delta}^{1}, \tilde{\Delta}$ are induced by the $\Delta_{n}$. When $i=0$, each term in the diagram is a set with distinguished base point (determined by the compatible sequence of base points $\left\{\alpha_{n}\right\},\left\{\alpha_{n}^{\prime}\right\}$ ) and exactness is defined in the usual way.

Lemma 7.2. If, for each $n$, the mapping $\Delta_{n}: \mathscr{F}\left(A_{n}, B\right) \rightarrow \mathscr{F}\left(\Delta B, \Delta A_{n}\right)$ is a weak equivalence, then the mapping

$$
\Delta: \mathscr{F}(A, B) \rightarrow \mathscr{F}(\Delta B, \Delta A)
$$

is a weak equivalence.

Proof. If $i>0$, it follows immediately from (7.1) that the mapping

$$
\Delta_{*}: \pi_{i}(\mathscr{F}(A,), \alpha) \rightarrow \pi_{i}\left(\mathscr{F}(\Delta B, \Delta A), \alpha^{\prime}\right)
$$

is an isomorphism. Suppose that $i=0$ and that we have $b_{1}, b_{2} \in \pi_{0}(\mathscr{F}(A, B))$ with $\Delta_{*} b_{1}=\Delta_{*} b_{2}$. It then follows from diagram (7.1) that $j_{*} b_{1}=j_{*} b_{2}=$ $\left\{\alpha_{n}\right\} \in \lim \pi_{0}\left(\mathscr{F}\left(A_{n}, B\right)\right)$. We use this sequence of base points as our distinguished points in the top row of (7.1) (with $i=0$ ) and $\Delta_{n} \alpha_{n}=\alpha_{n}^{\prime}$ as the distinguished base points in the bottom row. By exactness, we can find $c_{1}, c_{2} \in$ $\lim ^{1} \pi_{1}\left(\mathscr{F}\left(A_{n}, B\right), \alpha_{n}\right)$ with $j_{*} c_{1}=b_{1}, j_{*} c_{2}=b_{2}$. But then $i_{*}^{\prime} \tilde{\Delta}^{1} c_{1}=i_{*}^{\prime} \tilde{\Delta}^{1} c_{2}$ which implies $c_{1}=c_{2}$. Thus $b_{1}=b_{2}$ and

$$
\Delta_{*}: \pi_{0}(\mathscr{F}(A, B)) \rightarrow \pi_{0}(\mathscr{F}(\Delta B, \Delta A))
$$

is injective. The proof of surjectivity is similar and is left to the reader.

We now proceed with the proof that

$$
\Delta_{n}: \mathscr{F}\left(A_{n}, B\right) \rightarrow \mathscr{F}\left(\Delta P, \Delta A_{n}\right)
$$

is a weak equivalence. We know by Lemma 6.12 that the fibration

$$
\pi: \mathscr{F}\left(A_{n}, B\right) \rightarrow \mathscr{F}\left(A_{n-1}, B\right)
$$

is induced from the fibration

$$
\mathscr{F}(R[x, y], B) \rightarrow \mathscr{F}(R[y], B)
$$


by the mapping

$$
f_{*}: \mathscr{F}\left(A_{n-1}, B\right) \rightarrow \mathscr{F}(R[y], B),
$$

where $\operatorname{deg} y=1+\operatorname{deg} x=1+\operatorname{deg} x_{n}$ and $f: R[y] \rightarrow A_{n-1}$ is given by $f(y)=$ $d x_{n}$. Furthermore $\mathscr{F}(R[x, y], B)$ is contractible (by Lemma 6.10) so the image of the mapping (7.4) is contained in a single component $X$ of $\mathscr{F}(R[y], B)$. Now, if $Z^{\prime}$ is a component of $\mathscr{F}\left(A_{n-1}, B\right)$, then $\pi^{-1} Z^{\prime} \subset \mathscr{F}\left(A_{n}, B\right)$ is empty unless

$$
f_{\#} Z^{\prime} \subset X \text {. }
$$

It follows that $\mathscr{F}\left(A_{n}, B\right)$ is the disjoint union of the $\pi^{-1} Z^{\prime}$ as $Z^{\prime}$ ranges over those components of $\mathscr{F}\left(A_{n-1}, B\right)$ satisfying (7.5).

In the same way, we see that the fibration

$$
\pi^{\prime}: \mathscr{F}\left(\Delta B, \Delta A_{n}\right) \rightarrow \mathscr{F}\left(\Delta B, \Delta A_{n-1}\right)
$$

is induced by the mapping

$$
(\Delta f)_{\#}: \mathscr{F}\left(\Delta B, \Delta A_{n-1}\right) \rightarrow \mathscr{F}(\Delta B, R[y])
$$

from the fibration

$$
\mathscr{F}(\Delta B, \Delta R[x, y]) \rightarrow \mathscr{F}(\Delta B, \Delta R[y]),
$$

where $\mathscr{F}(\Delta B, \Delta[R x, y])$ is contractible since $\Delta R[x, y]$ is (by Lemma 6.10). Thus, if $X^{\prime}$ is the component of $\mathscr{F}(\Delta B, \Delta R[y])$ containing the image of the mapping (7.7), then $\mathscr{F}\left(\Delta B, \Delta A_{n}\right)$ is the disjoint union of $\left(\pi^{\prime}\right)^{-1} Z$; where $Z^{\prime}$ ranges over the components of $\mathscr{F}\left(\Delta B, \Delta A_{n-1}\right)$ satisfying $(\Delta f)_{\#} Z^{\prime} \subset X$.

We can now apply Proposition 6.6 to the fibrations (7.3) and (7.6) obtaining the diagram of exact sequences

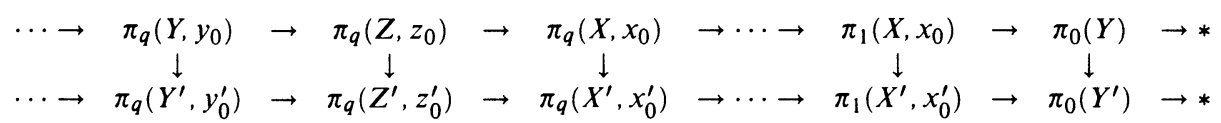

Here $X$ is the image of the mapping (7.4), $Z$ is any component of $\mathscr{F}\left(A_{n-1}, B\right)$ mapping into $X$ under the mapping $f_{\#}$ and $Y=\pi^{-1} Z \subset \mathscr{F}\left(A_{n}, B\right)$. Similarly, $X^{\prime}$ is the image of (7.7), $Z^{\prime}$ is any component of $\mathscr{F}\left(\Delta B, \Delta A_{n-1}\right)$ mapping into $X^{\prime}$ under the mapping $(\Delta f)_{\#}$, and $Y^{\prime}=\left(\pi^{1}\right)^{-1} Z^{\prime} \subset \mathscr{F}\left(\Delta B, \Delta A_{n}\right)$.

Using Lemma 7.2, the 5-Lemma, and induction on $n$, we have the following.

Lemma 7.8. If the mapping

$$
\Delta: \mathscr{F}(A, B) \rightarrow \mathscr{F}(\Delta B, \Delta A)
$$

is a weak equivalence for $A=R[y]$, then it is a weak equivalence for any $A$ which is FNF.

To prove that $\Delta: \mathscr{F}(R[y], B) \rightarrow \mathscr{F}(\Delta B, \Delta R[y])$ is a weak equivalence, we need the following. 
Lemma 7.9. The mapping

$$
\Delta_{*}: \pi_{0}(\mathscr{F}(R[y], B)) \rightarrow \pi_{0}(\mathscr{F}(\Delta B, \Delta R[y])
$$

is a bijection for $y$ of arbitrary degree. Furthermore, if degree $y=0$, then

$$
\Delta_{*}: \pi_{j}(\mathscr{F}(R[y], B), \alpha) \rightarrow \pi_{j}\left(\mathscr{F}(\Delta B, \Delta R[y]), \alpha^{\prime}\right)
$$

is an isomorphism for all $j>0$.

Corollary 7.10. The mapping

$$
\Delta: \mathscr{F}(R[y], B) \rightarrow \mathscr{F}(\Delta B, \Delta R[y])
$$

is a weak equivalence.

Proof of Corollary 7.10. Consider the diagram of fibrations

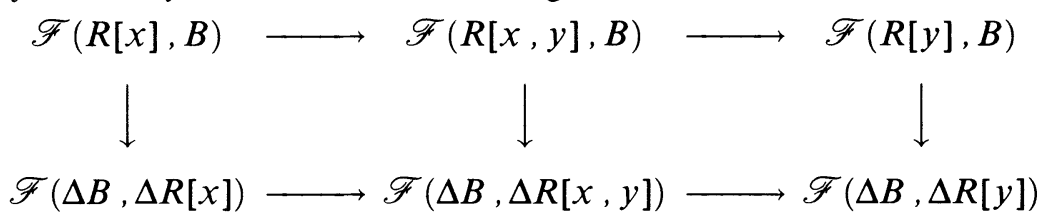

where $d x=y$ in $R[x, y]$. Since the total space of each of the above fibrations is contractible (by Lemma 6.10), the homotopy exact sequences of these fibrations reduce to

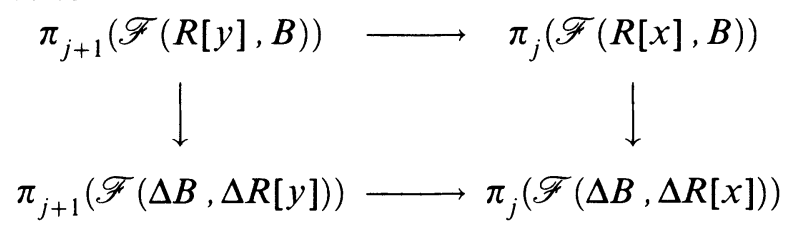

An easy induction using Lemma 7.9 gives the required result.

It now follows that the first assertion of Theorem 2.20 will be proved once we prove Lemma 7.9. Indeed, the arguments that we have developed in this section up to this point can be modified by making the obvious substitutions so as to deal with the second and third assertions of Theorem 2.20. As a result, Theorem 2.20 will be proved once we prove Lemma 7.9 and the following lemma.

Lemma 7.11. The mappings

$$
\begin{gathered}
\gamma_{*}: \pi_{0}\left(\mathscr{F}(R[y], \mathscr{A}(X)) \rightarrow \pi_{0}(\mathscr{F}(X, \Delta R[y]),\right. \\
f_{*}: \pi_{0}(\mathscr{F}(R[y], B)) \rightarrow \pi_{0}(\mathscr{F}(R[y], C))
\end{gathered}
$$

are bijections for $y$ of arbitrary degree. Furthermore, if degree $y=0$, then

$$
\begin{gathered}
\gamma_{*}: \pi_{j}(\mathscr{F}(R[y], \mathscr{A}(X)), \alpha) \rightarrow \pi_{j}\left(\mathscr{F}(X, \Delta R[y]), \alpha^{\prime}\right), \\
\left.\left.f_{*}: \pi_{j}(\mathscr{F}(R[y], B)), \alpha\right) \rightarrow \pi_{j}(\mathscr{F}(R[y], C)), \alpha^{\prime}\right)
\end{gathered}
$$

are isomorphisms for all $j>0$.

The proofs of Lemmas 7.9 and 7.11 involve the same ideas so we present them together. We begin by defining an isomorphism

$$
\xi: \pi_{0}(\mathscr{F}(R[y], B)) \rightarrow H_{n}(B),
$$


where $n=\operatorname{dim} y$. (Note: Both $\mathscr{F}(R[y], B)$ and $\mathscr{F}(\Delta B, \Delta R[y])$ are simplicial groups so $\pi_{0}(\mathscr{F}(R[y], B))$ and $\pi_{0}(\mathscr{F}(\Delta B, \Delta R[y]))$ are groups.)

By definition,

$$
\mathscr{F}(R[y], B)_{0}=\langle R[y], B\rangle=Z_{n}(B),
$$

where $Z_{n}(B)$ is the group of $n$-cycles in $B$. It follows that we have an epimorphism

$$
\xi: \mathscr{F}(R[y], B) \rightarrow H_{n}(B) .
$$

Suppose $z \in \mathscr{F}(R[y], B)_{0}$ is in the same component as 0 . Then there is an element $w \in \mathscr{F}(R[y], B)_{1}=Z_{n}\left(\Omega_{1} \otimes B\right)$ with $\partial_{0} w=z, \partial_{1} w=0$. If we define $v:\left(\Omega_{1} \otimes B\right)^{n} \rightarrow B^{n-1}$ by $v(a \otimes b)=0 \quad$ if $a \in \Omega_{1}^{0}, b \in B^{n}$, and if $b \in B^{n-1}$,

$$
v\left(a d t_{1} \otimes b\right)=\left(\int_{0}^{1} a\left(t_{1}\right) d t_{1}\right) b
$$

then $d v+v d=\partial_{0}-\partial_{1}$ so $d v(w)=z$. Hence $\xi$ induces an epimorphism

$$
\xi: \pi_{0}(\mathscr{F}(R[y], B)) \rightarrow H_{n}(B) .
$$

To show that $\xi$ is a monomorphism, suppose $z=d v$ for some $v \in B^{n-1}$. Then, if $\bar{v} \in\left(\Omega_{1} \otimes B\right)_{n}$ is given by

$$
\bar{v}=t_{1} \otimes z+d t_{1} \otimes v,
$$

we have $\partial_{0} \bar{v}=z, \partial_{1} \bar{v}=0$ and $z=0$ in $\pi_{0}(\mathscr{F}(R[y], B))$. It follows that $\xi$ is an isomorphism.

Now, if $f: B \rightarrow C$ is a DG algebra mapping, we have a commutative diagram

$$
\begin{array}{ccc}
\pi_{0}(\mathscr{F}(A, B)) & \stackrel{f_{*}}{\longrightarrow} \pi_{0}(\mathscr{F}(A, C)) \\
\xi \downarrow \simeq & \xi \downarrow \simeq \\
H_{n}(B) & \longrightarrow & H_{n}(C) .
\end{array}
$$

If $f_{*}: H_{*}(B) \rightarrow H_{*}(C)$ is an isomorphism, then

$$
f_{*}: \pi_{0}(\mathscr{F}(A, B)) \rightarrow \pi_{0}(\mathscr{F}(A, C))
$$

is an isomorphism and the second of the four assertions of Lemma 7.11 is proved.

We next define an isomorphism

$$
\xi^{\prime}: \pi_{0}(\mathscr{F}(X, \Delta R[y])) \rightarrow H_{n}(\mathscr{A}(X))
$$

for any simplicial space $X$. By definition,

$$
\begin{aligned}
\mathscr{F}(X, \Delta(R[y]))_{q} & =\left(X \times \Delta[q], Z_{n}(\Delta)\right) \\
& =Z_{n}(\mathscr{A}(X) \times \Delta[q]) \\
& =Z_{n}(\mathscr{A}(X)) \quad \text { if } q=0 .
\end{aligned}
$$


Let

$$
\xi^{\prime}: \pi_{0}(\mathscr{F}(X, \Delta(R[y]))) \rightarrow H_{n}(\mathscr{A}(X))
$$

be given by

$$
\xi^{\prime}\{z\}=z+B_{n}(\mathscr{A}(X)) .
$$

We show that $\xi^{\prime}$ is well defined and an isomorphism.

Recall that in $\S 5$, we defined $\Omega^{p}\left(\Delta^{q} \times I\right)$ to be the $p$ forms on $\Delta^{q} \times I$ and we defined

by

$$
\mu: \Omega^{p}\left(\Delta^{p} \times I\right) \rightarrow \Omega_{q}^{p-1}
$$

$$
\mu(w)=(-1)^{p-1} \int_{0}^{1} w_{2}(t) d t
$$

where $w=w_{1}(t)+w_{2}(t) d t$. Note that we may view the elements of $\mathscr{A}^{p}(\Delta[q] \times \Delta[1])$ as continuous, piecewise smooth $p$-forms on $\Delta^{q} \times I$ and the formula for $\mu$ makes sense on such forms and gives a map

$$
\mu: \mathscr{A}^{p}(\Delta[q] \times \Delta[1]) \rightarrow \mathscr{A}^{p-1}(\Delta[q]) .
$$

Furthermore, we have $d \mu+\mu d=i_{1}^{*}-i_{0}^{*}$, where $i_{0}, i_{1}: \Delta[q] \rightarrow \Delta[q] \times \Delta[1]$ are the inclusions.

To see that $\xi^{\prime}$ is well defined, suppose $z_{0}$ and $z_{1}$ are elements of $Z_{n}(\mathscr{A}(X))$ and $z_{i}=\partial_{i} v$, where $v \in Z_{n}(\mathscr{A}(X \times \Delta[1]))$. Let $\bar{v} \in \mathscr{A}^{n-1}(X)$ be given by

$$
\bar{v}(x)=\mu\left(t_{x} \times \mathrm{id}_{\Delta[1]}\right)^{*} v
$$

for $x \in X_{q}$, where $t_{x}: \Delta[q] \rightarrow X$ with $t_{x}(0, \ldots, q)=x$. Then

$$
\begin{aligned}
d \bar{v}(x) & =\left(i_{1}^{*}-i_{0}^{*}-\mu d\right)\left(t_{x} \times \mathrm{id}_{\Delta[1]}\right)^{*} v \\
& =\left(i_{1}^{*}-i_{0}^{*}\right)\left(t_{x} \times \mathrm{id}_{\Delta[1]}\right)^{*} v \\
& =\left(\partial_{0} v-\partial_{1} v\right)(x)=z_{0}(x)-z_{1}(x)
\end{aligned}
$$

so $\xi^{\prime}$ is well defined.

Clearly $\xi^{\prime}$ is an epimorphism; we show it is a monomorphism. Suppose $z=d v, v \in \mathscr{A}^{n-1}(\Delta(B))$. Let $w \in Z_{n}(\mathscr{A}(X \times \Delta[1]))$ be given as follows: If $p_{1}$ and $p_{2}$ are the projectives of $X \times \Delta[1]$ onto the factors, then

$$
w(x)=\left(d\left(p_{2}^{*} t_{1}\right)\right) p_{1}^{*} v(x)+\left(p_{2}^{*} t_{1}\right)\left(p_{1}^{*} z\right)(x) .
$$

It is easily checked that $d w=0, \partial_{0} w=0$, and $\partial_{1} w=z$. Thus $\xi^{\prime}$ is an isomorphism.

It follows directly from the definitions that the diagrams

$$
\begin{array}{ccc}
\pi_{0}(\mathscr{F}(R[y], B)) & \stackrel{\Delta_{0}}{\longrightarrow} & \pi_{0}(\mathscr{F}(\Delta B, \Delta R[y])) \\
\xi\rfloor \simeq & \xi^{\prime}\lfloor\simeq \\
H_{n}(B) & \stackrel{i_{*}}{\longrightarrow} & H_{n}(\mathscr{A} \Delta B)
\end{array}
$$


and

$$
\begin{array}{ccc}
\pi_{0}(\mathscr{F}(R[y], A(X))) & \stackrel{\gamma}{\longrightarrow} & \pi_{0}(\mathscr{F}(X, \Delta R[y])) \\
\xi \downarrow \simeq & & \xi^{\prime} \downarrow \simeq \\
H_{n}(\mathscr{A}(X)) & \stackrel{\text { id }}{\longrightarrow} & H_{n}(\mathscr{A}(X))
\end{array}
$$

are commutative, where $i_{*}: H_{n}(B) \rightarrow H_{n}(\mathscr{A}(\Delta B))$ is the canonical mapping defined in $\S 2$. Since $B$ is FNF, $i_{*}$ is an isomorphism by Proposition 2.8. The first assertions of both Lemmas 7.9 and 7.11 are an immediate consequence of these diagrams.

We now prove the remaining assertions of Lemmas 7.9 and 7.11, namely that certain mappings between simplicial spaces defined in terms of $R[y]$ induce isomorphisms on homotopy in positive dimensions if degree $y=0$. In fact, we prove that all of these homotopy groups vanish.

Assume degree $y=0$. By definition,

$$
\mathscr{F}(R[y], B)_{q}=\left\langle R[y], \Omega_{q} \otimes B\right\rangle=Z_{0}\left(\Omega_{q} \otimes B\right) .
$$

It is easy to see that $d(f \otimes b)=0$ for $f \in \Omega_{q}^{0}, b \in B^{0}$ if and only if $d f=0$ and $d b=0$. Thus,

$$
\mathscr{F}(R[y], B)_{q}=H_{0}(B)
$$

for all $q \geq 0$ and all face and degeneracy mappings are the identity. Therefore, $\pi_{j}(\mathscr{F}(R[y], B), \alpha)=0$ for all $j>0$.

Similarly, $\Delta R[y]_{q}=R$ for all $q \geq 0$ and all face and degeneracy mappings in $\Delta R[y]$ are the identity. It follows that, for any $Z \in \Delta \mathscr{T}$,

$$
\mathscr{F}(Z, \Delta R[y])_{q}=\langle\Delta[q] \times Z, \Delta R[y]\rangle
$$

consists of mappings that are constant on components; that is, all continuous mappings of $\pi_{0}(Z)$ into $R$ and all face and degeneracy mappings the identity. Again, $\pi_{j}(\mathscr{F}(Z, \Delta R[y]))=0$ for $j>0$ and Theorem 2.20 is proved.

\section{The Serre sPeCtral Sequence for continuous cohomology}

Let $B \times{ }_{\tau} F$ be a twisted cartesian product with group $G$. If $\bar{\tau}=\bar{\tau}_{q}: B_{q} \rightarrow$ $G_{q-1}$ is defined by

$$
\bar{\tau}(b)=s_{0}^{q-1} \partial_{1}^{q-1} \tau(b)
$$

for $b \in B_{q}$, one easily checks that it is a twisting function if $G_{0}=\pi_{0}(G)$. Define

$$
\lambda: G_{0} \times C^{q}(F ; R) \rightarrow C^{q}(F ; R)
$$

by

$$
(\lambda(g, u))(y)=u\left(\left(s_{0}^{q} g\right) y\right) .
$$

This mapping induces a continuous mapping

$$
\lambda: G_{0} \times H^{q}(F) \rightarrow H^{q}(F) .
$$


We define the twisted continuous cohomology groups of $B$ with coefficients in $H^{q}(F), \quad H^{p}\left(B ; H^{q}(F)_{\tau}\right)$, to be the homology of the cochain complex $C^{p}\left(B ; H^{q}(F)\right)=$ continuous functions from $B_{p}$ to $H^{q}(F)$ vanishing on degeneracies with

$$
\delta: C^{p}\left(B ; H^{q}(F)\right) \rightarrow C^{p+1}\left(B ; H^{q}(F)\right)
$$

given by

$$
(\delta u)(b)=\lambda\left(\partial_{0}^{p-1} \bar{\tau}(b), u\left(\partial_{0} b\right)\right)+\sum_{i=1}^{p+1}(-1)^{i} u\left(\partial_{i} b\right) .
$$

Recall that for $X \in \Delta \mathscr{T}$ the $n$-skeleton of $X$ is the smallest subsimplicial space $X^{(n)}$ of $X$ containing $X_{n}$. Let $E=B \times{ }_{\tau} F$ and $\mathscr{F}_{p} E=\pi^{-1}\left(B^{(p)}\right)$. Following the notation of [12], we define the Serre spectral sequence of $E=$ $B \times{ }_{\tau} F$ by

$$
\begin{aligned}
A^{p, q}= & \left\{u \in C^{p+q}(E) \mid u\left(\mathscr{F}_{p-1} E\right)=0\right\}, \\
E_{r}^{p, q}= & A^{p, q} \cap \delta^{-1}\left(A^{p+r, q-r+1}\right) / A^{p+1, q-1} \cap \delta^{-1}\left(A^{p+r, q-r+1}\right) \\
& +A^{p, q} \cap \delta A^{p-r+1, q+r-2}, \\
d_{r}\{u\}= & \{\delta u\}, \\
D^{p, q}= & \text { image } H_{p+q}\left(A^{p}\right) \rightarrow H_{p+q}\left(C^{*}(E)\right),
\end{aligned}
$$

where $A^{p}=\sum_{q \geq 0} A^{p, q}$.

Elementary quotient ring manipulations yield the following (see [12] for proofs).

Theorem 8.1. $\left\{E_{r}, d_{r}\right\}$ is a multiplicative spectral sequence (with respect to the usual cup products) with $E_{r}^{p, q} \approx D^{p, q} / D^{p+1, q-1}$ for $r$ sufficiently large.

We next turn to the calculation of $E_{2}$, which requires some hypotheses on $F$. The main difficulty is in proving that the cohomology $H^{*}(B \times F)$ of the product is isomorphic to $H^{*}\left(B ; H^{*}(F)\right)$. We define $C^{*}(F)$ to be splittable if the usual arguments yield this result, namely:

Definition 8.2. A differentiable graded topological vector space $C$ is splittable if the mappings

$$
\begin{gathered}
\delta: C^{q} \rightarrow B^{q+1}=\left\{\delta u: u \in C^{q}\right\}, \\
\eta: Z^{q}=\left\{u \in C^{q}: \delta u=0\right\} \rightarrow H_{q}(C)
\end{gathered}
$$

have continuous (not necessarily linear) sections for all $q \geq 0$. A simplicial space $X$ is splittable if $C^{*}(X ; R)$ is splittable.

We develop some of the properties of this notion at the end of this section.

Theorem 8.3. If $F$ is splittable and $G_{0}=\pi_{0}(G)$, then the usual map yields a ring isomorphism

$$
E_{2}^{p, q} \approx H^{p}\left(B ; H^{q}(F)_{\tau}\right)
$$


Proof. The proof of this result is accomplished by a series of lemmas. Let $E_{r}^{p, q}$ and $\bar{E}_{r}^{p, q}$ be the spectral sequences associated to $E=B \times{ }_{\tau} F$ and $\bar{E}=E \times{ }_{\bar{\tau}} F$ respectively. Note that $A^{p, q}=\bar{A}^{p, q}$. We first prove that $E_{r}^{p, q}=\bar{E}_{r}^{p, q}$ for $r \leq 2$.

Let $V_{j}: B_{q} \times F_{q} \rightarrow B_{q} \times F_{q}, 1 \leq j \leq q-1$, be given by

$$
V_{j}(b, y)=\left(s_{j} \partial_{0} b,\left(s_{0}^{j} \partial_{1}^{j-1} \tau(b)\right) s_{j} \partial_{0} y\right)
$$

and $V: C^{n}(E) \rightarrow C^{n}(E)$ by

$$
V u=\sum_{j=1}^{q-1}(-1)^{j} u V_{j} .
$$

Lemma 8.4. The map $V$ is continuous and satisfies $V\left(A^{p}\right) \subset A^{p+1}$ and

$$
\left(\delta_{\tau} V-V \delta_{\tau}-\delta_{\tau}+\delta_{\bar{\tau}}\right)\left(A^{p}\right) \subset A^{p+2},
$$

where $\delta_{\tau}: C^{n}(E) \rightarrow C^{n+1}(E)$ and $\delta_{\bar{\tau}}: C^{n}(\bar{E}) \rightarrow C^{n+1}(\bar{E})$ are the usual differentials.

Proof. Suppose $u \in A^{p},(b, y) \in\left(B \times_{\tau} F\right)_{q}, b \in B^{(p)}$. We want to show that $V(u)(b, y)=0$. If $\partial_{0} b \in B^{(p-1)}, s_{j} \partial_{0} b \in B^{(p-1)}$ and hence, $V(u)(b, y)=0$. Suppose $\partial_{0} b \notin B^{(p-1)}$. Then $b=s_{0} b^{\prime}$ and $V_{j}(b, y)=s_{j}\left(\partial_{0} b, \partial_{0} y\right)$, which is degenerate, and hence $V(u)(b, y)=0$.

Now suppose $b \in B^{(p+1)}$. We show that

$$
\left(\delta_{\tau} V-V \delta_{\tau}-\delta_{\tau}+\delta_{\bar{\tau}}\right)(u)(b, y)=0 .
$$

First note that

$$
\left(\delta_{\tau}-\delta_{\bar{\tau}}\right)(u)(b, y)=u\left(\partial_{0} b, \tau(b) \partial_{0} f\right)-u\left(\partial_{0} b, s_{0}^{q-1} \partial_{1}^{q-1} \tau(b) \partial_{0} f\right) .
$$

It is straightforward to verify that for $i>0$

$$
\begin{aligned}
\partial_{i} V_{j}(b, y) & = \begin{cases}V_{j-1}\left(\partial_{i+1} b, \partial_{i+1}, y\right), & i<j<q, \\
\left(\partial_{0} b, s_{0}^{j-1} \partial_{1}^{j-1} \tau(b) \partial_{0} y\right), & i=j, \\
\left(\partial_{0} b, s^{j} \partial_{1}^{j} \tau(b) \partial_{0} y\right), & i=j+1, \\
V_{j}\left(\partial_{i} b, \partial_{i} y\right), & i>j+1,\end{cases} \\
\partial_{0} V_{j}(b, y) & =\left(s_{j-1} \partial_{0} \partial_{0} b,\left(s_{j-1} \tau\left(\partial_{0} b\right)\right)\left(\partial_{0}^{j-1} \partial_{1}^{j-1} \tau(b)\right)\left(s_{j-1} \partial_{0} \partial_{0} y\right)\right), \\
V_{j}\left(\partial_{j+1} b, \partial_{j+1} y\right) & =s_{j}\left(\partial_{0} \partial_{j+1} b,\left(s_{0}^{j-1} \partial_{1}^{j} \tau(b)\right) \partial_{0} \partial_{j+1} y\right), \\
V_{j}\left(\partial_{1} b, \partial_{1} y\right) & =\left(s_{j} \partial_{0} \partial_{0} b,\left(s_{0}^{j} \partial_{1}^{j+1} \tau\left(\partial_{0} b\right)\right)\left(s_{0}^{j} \partial_{1}^{j-1} \partial_{0} \tau(b)\right) s_{0} \partial_{0} \partial_{0} y\right), \\
V_{j}\left(\partial_{0} b, \tau(b) \partial_{0} y\right) & =\left(s_{j} \partial_{0} \partial_{0} b,\left(s_{0}^{j} \partial_{1}^{j-1} \tau\left(\partial_{0} b\right)\right)\left(s_{j} \partial_{0} \tau(b)\right)\left(s_{j} \partial_{0} \partial_{0} y\right)\right) .
\end{aligned}
$$

Substituting these in (8.5), all the terms cancel, except possibly those above starting with $s_{j} \partial_{0} \partial_{0} b, b \in B^{(p+1)}$. Hence, $s_{j} \partial_{0} \partial_{0} b \in B^{p-1}$ unless $b=s_{0} b^{\prime}$, in which case $\tau(b)=e$, the last two terms cancel, and $\partial_{0} V_{j}(b, f)$ is degenerate. 
Lemma 8.6. For $r \leq 2, E_{r}^{p, q}=\bar{E}_{r}^{p, q}$, where $E_{r}$ and $\bar{E}_{r}$ are the spectral sequences associated to $B \times{ }_{\tau} F$ and $B \times{ }_{\bar{\tau}} F$ respectively.

Proof. As mentioned earlier, $A^{p}=\bar{A}^{p}$. One easily checks, using Lemma 8.4, that $d_{0}=\bar{d}_{0}$ and $d_{1}=\bar{d}_{1}$. Thus

$$
E_{2}=H_{*}\left(E_{1}, d_{1}\right)=H_{*}\left(\bar{E}_{1}, \bar{d}_{1}\right)=\bar{E}_{2} \text {. }
$$

Theorem 8.7. Let $\left\{E_{r}^{p, q}, d_{r}\right\}$ be the spectral sequence for $E=B \times{ }_{\tau} F$. Then, if $F$ is splittable, we have $E_{2}^{p, q} \simeq H^{p}\left(B ; H^{q}(F)_{\tau}\right)$.

Proof. We begin with a digression. Let $X=\left\{X_{p, q}\right\}$ be a bisimplicial space with face and degeneracy mappings

$$
\begin{gathered}
\partial_{i}^{\prime}: X_{p, q} \rightarrow X_{p-1, q}, \quad \partial_{i}^{\prime \prime}: X_{p, q} \rightarrow X_{p, q-1}, \\
s_{i}^{\prime}: X_{p, q} \rightarrow X_{p+1, q}, \quad s_{i}^{\prime \prime}: X_{p, q} \rightarrow X_{p, q+1} .
\end{gathered}
$$

The diagonal of $X$ is the simplicial space $\hat{X}$, where $\hat{X}_{p}=X_{p, p}, \partial_{i}=\partial_{i}^{\prime} \partial_{i}^{\prime \prime}=$ $\partial_{i}^{\prime \prime} \partial_{i}^{\prime}$, and $s_{j}=s_{j}^{\prime} s_{j}^{\prime \prime}=s_{j}^{\prime \prime} s_{j}^{\prime}$. Let $C(X)=\left(C^{p, q}, d^{\prime}, d^{\prime \prime}\right)$ be the double complex defined by

$$
\begin{gathered}
C^{p, q}=\left\{u \in\left(X_{p, q}, R\right): u \circ s_{i}^{\prime}=u \circ s_{j}^{\prime \prime}=0,0 \leq i \leq p-1,0 \leq j \leq q-1\right\}, \\
d^{\prime} u=\sum_{i=0}^{p+1}(-1)^{i} u \circ \partial_{i}^{\prime}, \quad d^{\prime \prime} u=\sum_{j=0}^{q+1}(-1)^{j} u \circ \partial_{j}^{\prime \prime}
\end{gathered}
$$

and denote by $C_{t}(X)$ the associated total complex. Define $T: C_{t}(X) \rightarrow C(\hat{X})$ by

$$
(T u)(x)=u\left(\left(\partial_{p+1}^{\prime}\right)^{q}\left(\partial_{0}^{\prime \prime}\right)^{p} x\right)
$$

for $u \in C^{p, q}, x \in \hat{X}_{p+q}$.

Lemma 8.8. There is a mapping $T^{\prime}: C(\hat{X}) \rightarrow C_{t}(X)$ and cochain homotopies $\varphi: C(\hat{X}) \rightarrow C(\hat{X}), \psi: C_{t}(X) \rightarrow C_{t}(X)$ with

$$
\delta \varphi+\varphi \delta=T T^{\prime}-\mathrm{id}, \quad \delta \psi+\psi \delta=T^{\prime} T-\mathrm{id} .
$$

Furthermore, $\Omega_{*}: H_{*}\left(C_{*}(X)\right) \rightarrow H_{*}(C(\hat{X})$ is an algebra isomorphism.

The proof of the first part of the lemma follows the same lines as the proof of Theorem 2.9 of Dold and Puppe [7]. To prove that $T$ preserves products, we need a variant of the cup- $i$ products.

For $u \in C^{p, q}(X), v \in C^{r, s}(X)$, define $u \cup_{1} v \in C^{p+q+r+s-1}(\hat{X})$ by

$$
\left(u \cup_{1} v\right)(x)=\sum_{j=p}^{p+q}(-1)^{(q-1)(r+1)} u\left(D_{j} x\right) v\left(E_{j} x\right) \text {, }
$$

where

$$
\begin{aligned}
& D_{j}=\left(\partial_{p+1}^{\prime}\right)^{q+r+s-1}\left(\partial_{0}^{\prime \prime}\right)^{p}\left(\partial_{j}^{\prime \prime}\right)^{r-1}\left(\partial_{p+q+r}^{\prime \prime}\right)^{s}, \\
& E_{j}=\left(\partial_{0}^{\prime}\right)^{j}\left(\partial_{j+r+1}^{\prime}\right)^{p+q+s-j-1}\left(\partial_{0}^{\prime \prime}\right)^{p+q+r-1} .
\end{aligned}
$$


The fact that $T_{*}$ is multiplicative now follows from

Lemma 8.9. If $u \cup_{1} v$ is defined as above, then

$$
\begin{aligned}
\delta\left(u \cup_{1} v\right)= & (\delta u) \cup_{1} v+(-1)^{p+q} u \cup_{1} \delta v \\
& +(-1)^{p+q+r}(T(u v)-T(u) T(v)) .
\end{aligned}
$$

The proof of this equation proceeds by first proving it in the special case where $X_{p, q}=Y_{p} \times Z_{q}, \quad Y$ and $Z$ simplicial sets. This case can be seen to follow from the commutativity of ordinary cup products as in Steenrod [20]. The general case now follows from the fact that given $x \in X_{p, q}$, there is a unique bisimplicial mapping

$$
f: \Delta[p] \times \Delta[q] \rightarrow X
$$

with $f((0, \ldots, p),(0, \ldots, q))=x$. The details are left to the reader.

Define a bisimplicial complex $X$ by $X_{p, q}=B_{p} \times F_{q}$ with

$$
\begin{aligned}
& \partial_{i}^{\prime}(b, y)= \begin{cases}\left(\partial_{0} b, s_{0}^{q} \tau\left(\partial_{2}^{p-1} b\right) y\right), & i=0, \\
\left(\partial_{i} b, y\right), & i>0,\end{cases} \\
& \partial_{i}^{\prime \prime}(b, y)=\left(b, \partial_{i} y\right), \\
& s_{i}^{\prime}(b, y)=\left(s_{i} b, y\right), \quad s_{i}^{\prime \prime}(b, y)=\left(b, s_{i} y\right),
\end{aligned}
$$

for $b \in B_{p} y \in F_{q}$. Note that $\hat{X}=B \times_{\bar{\tau}} F$. Define a filtration of $C_{t}(X)$ by

$$
\tilde{A}^{p}=\sum_{j \geq p} \sum_{q \geq 0} C^{j, q}
$$

and let $\tilde{E}_{r}$ be the associated spectral sequence. Then it is easy to check that the mappings of Lemma 8.8 are filtration preserving so that $T$ induces an isomorphism $\tilde{E}_{2}^{p, q} \simeq \bar{E}_{2}^{p, q}$ and we need only prove that

$$
\tilde{E}_{2}^{p, q} \simeq H^{p}\left(B ; H^{q}(F)_{\tau}\right) .
$$

Now, $\tilde{E}_{1}$ is the homology of $C_{t}(X)$ with respect to the differential $d_{2}: C^{p, q}$ $\rightarrow C^{p, q+1}$. Since $F_{q}$ is compactly generated for all $q \geq 0$, we can identify

$$
C^{p, q}=C^{p}\left(B ; C^{q}(F)\right)
$$

(see proof of Theorem 5.6 in [21]) and $\delta_{2}$ is induced from the coboundary operator on $C^{*}(F)$. We must show that

$$
\frac{\operatorname{ker} d_{2}: C^{p, q} \rightarrow C^{p, q+1}}{\operatorname{Im} d_{2}: C^{p, q-1} \rightarrow C^{p, q}} \simeq C^{p}\left(B ; H^{q}(F)\right) .
$$

To begin with, the image of the mapping

$$
d_{2}: C^{p}\left(B ; C^{q-1}(F)\right) \rightarrow C^{p}\left(B ; C^{q}(F)\right)
$$


is clearly contained in $C^{p}\left(B ; B^{q}(F)\right)$. Since $F$ is splittable, $\delta: C^{q-1}(F) \rightarrow$ $B^{q}(F)$ has a continuous section and it follows that

$$
d_{2} C^{p}\left(B ; C^{q-1}(F)\right)=C^{p}\left(B ; B^{q}(F)\right) .
$$

Next, the mapping

$$
C^{p}\left(B ; Z^{q}(F)\right) \rightarrow C^{p}\left(B ; H^{q}(F)\right)
$$

clearly has kernel $C^{p}\left(B ; B^{q}(F)\right)$ and the fact that $F$ is splittable again implies that this mapping is onto. This completes the proof of Theorem 8.3.

We conclude this section with three results concerning splittable spaces.

Proposition 8.10. If $C$ is a splittable differential graded topological vector space, then $H_{q}(C)$ is Hausdorff for all $q \geq 0$.

Proof. Let $t: H_{q}(C) \rightarrow Z_{q}$ be a section for $\eta: Z_{q} \rightarrow H_{q}(C)$. Then, for $x \neq y$ in $H_{q}(C)$, let $U$ and $V$ be disjoint neighborhoods of $t(x), t(y)$ in $Z_{q}$. Then $t^{-1} U$ and $t^{-1} V$ are disjoint neighborhoods of $x$ and $y$ in $H_{q}(C)$.

Definition 8.11. Let $C, \bar{C}$ be differential graded topological spaces. We say $\bar{C}$ is a retract of $C$ if there are continuous linear mappings

$$
\alpha: \bar{C} \rightarrow C, \quad \beta: C \rightarrow \bar{C}
$$

preserving differentials and continuous linear $D: \bar{C}^{q} \rightarrow \bar{C}^{q-1}, q>0$, with $\beta \alpha-\mathrm{id}=\bar{\delta} D+D \bar{\delta}$.

Proposition 8.12. If $C$ is splittable and $\bar{C}$ is a retract of $C$, then $\bar{C}$ is splittable. Proof. Let $s: B^{q} \rightarrow C^{q+1}, t: H^{q} \rightarrow Z^{q}$ be the mappings which exist because $C$ is splittable. A straightforward computation shows that the mappings

$$
\bar{s}: \bar{B}^{q} \rightarrow \bar{C}^{q+1}, \quad \bar{t}: \bar{H}^{q} \rightarrow \bar{Z}^{q}
$$

given by $\bar{s}=\beta s \alpha+D, \bar{t}=\beta t \alpha_{*}$ satisfy the conditions necessary to make $\bar{C}$ splittable.

Proposition 8.13. Let $X$ be a simplicial space such that $X_{q}$ is locally compact and countable at infinity for all $q \geq 0$. Then $C^{*}(X ; R)$ is splittable.

Proof. If $X_{q}$ is locally compact and countable at infinity, the space $\left(X_{q}, R\right)$ of continuous functions from $X_{q}$ to $R$ is a Fréchet space (by (4.7)). Since $C^{q}(X ; R)$ is a closed subspace of $\left(X_{q}, R\right)$, it is also a Fréchet space (by (4.5)). Proposition 8.13 now follows from (4.8).

\section{Proof of Lemma 2.8}

We wish to prove that if $A$ is FNF, then

$$
i_{*}: H_{*}(A) \rightarrow H_{*}(\mathscr{A}(\Delta A))
$$

is an isomorphism. Choose generators for $A$ so that $A=\bigcup A_{n}, A_{0}=R$, $A_{n}=A_{n-1}\left[x_{n}\right]$ as in Definition 2.5. Then, for fixed $p$ and $q, A^{p}=A_{n}^{p}$ and 
$\Delta(A)_{q}=\Delta\left(A_{n}\right)_{q}$ for $n$ sufficiently large so we can assume $A$ finitely generated. The proof now proceeds by induction on $n$. We first prove Lemma 2.8 for $n=1$, that is, when $\Delta A$ is a differential form version of $K(R, m)$. We begin with the easier task of determining $H^{*}(K(R, m))$.

Lemma 9.1. There is an isomorphism $H^{*}(K(R, 1)) \simeq R[x]$, where degree $x=1$. Proof. Sending $u \in Z^{1}(\Delta[q] ; R)$ into $(u(0,1), u(1,2), \ldots, u(q-1, q)) \in R^{q}$ defines an isomorphism $K(R, 1)_{q} \simeq R^{q}$ such that

$$
\partial_{i}\left(a_{1}, \ldots, a_{q}\right)= \begin{cases}\left(a_{2}, \ldots, a_{q}\right), & i=0, \\ \left(a_{1}, \ldots, a_{i+1}+a_{i}, \ldots, a_{q}\right), & 0<i<q, \\ \left(a_{1}, \ldots, a_{q-1}\right), & i=q .\end{cases}
$$

It follows that $H^{*}(K(R, 1))$ is the classical continuous cohomology of $R$ as a Lie group. It now follows from the Van Est theorem [24] that $H^{*}(K(R, 1))$ is the Lie algebra cohomology of the Lie algebra $R$ which is clearly $R[x]$.

Our next result proves Theorem 2.3.

Lemma 9.2. The algebra $C^{*}(K(R, n), R)$ is splittable and $H^{*}(K(R, n)) \simeq R[x]$ where degree $x=n$.

Proof. We prove this result by induction on $n$. The usual Serre spectral sequence argument applied to the TCP

$$
K(R, n) \subset C^{n}(R) \rightarrow K(R, n+1)
$$

(see Lemma 6.9) yields the desired result if we can show that the Serre spectral sequence is applicable. In going from $n$ to $n+1$, we need to know that $C^{*}(K(R, n))$ is splittable. Assume Lemma 8.2 is true for $n-1$ and hence, by the Serre spectral sequence, $H^{*}(K(R, n))$ is isomorphic to $R[x]$. We show $B^{q}(K(R, n)) \subset C^{q}(K(R, n))$ is closed. By hypothesis, the inclusion $K(Z, n) \subset$ $K(R, n)$ induces an isomorphism,

$$
H^{q}(K(R, n)) \rightarrow H^{q}(K(Z, n)) \stackrel{\simeq}{\rightarrow} \operatorname{Hom}\left(H_{q}(K(Z, n), R), R\right) .
$$

Hence for any $q$ for which these groups are nonzero, there is a chain $c_{q} \in C_{*}(K(Z, n))$ such that evaluation on $c_{q}$ gives a continuous isomorphism $H^{q}(K(R, n)) \rightarrow R$. Then $B^{q}$ is closed because it is the kernel of the continuous map of $Z^{n}(K(R, n))$ to $R$ given by evaluation on $c_{q}$.

For each $q \geq 0, K(R, n)_{q}$ is a Euclidean space and it follows from (4.7) that $\left(K(R, n)_{q}, R\right)$ is a Fréchet space. Thus, using (4.5) and (4.6), $C^{q}(K(R, n), R)$, $Z^{q}(K(R, n), R), B^{q}(K(R, n), R)$ and $H^{q}(K(R, n))$ are Fréchet spaces. The fact that $C^{*}(K(R, n), R)$ is a splittable algebra now follows from (4.8).

We now prove Lemma 2.8 for $A=R[y]$.

Lemma 9.3. The algebra $\mathscr{A} \Delta(R[y])$ is splittable and

$$
i_{*}: H^{*}(R[y]) \simeq R[y] \rightarrow H^{*}(\mathscr{A} \Delta(R[y]))
$$

is an isomorphism. 
Proof. We begin with the second assertion of the lemma. Suppose degree $y=$ $n$. Then the mappings $\varphi$ and $\psi$ of Lemma 5.5 give mappings

$$
K(R, n) \stackrel{\varphi}{\rightarrow} \Delta R[y] \stackrel{\psi}{\rightarrow} K(R, n)
$$

with $\psi \varphi=$ identity. We define a homotopy

$$
F: Z_{n}(\Omega) \times \Delta[1] \rightarrow Z_{n}(\Omega)
$$

between $\varphi \psi$ and the identity as follows. Let $s \in \Delta[1]_{q}$ and $w \in \Omega_{q}^{n}$ with $d w=0$. Then $s$ determines a unique simplicial mapping $\tilde{s}: \Delta[q] \rightarrow \Delta[1]$ with $\tilde{s}(0,1, \ldots, q)=s$ and thus a linear mapping $\hat{s}: \Delta^{q} \rightarrow \Delta^{1}$. Let $f: \Delta^{q} \rightarrow \Delta^{q} \times \Delta^{q}$ be the mapping id $\times \hat{s}$ and define

$$
F(w, s)=f^{*}\left(\left(p_{1}^{*} w\right)\left(p_{2}^{*} t_{1}\right)+\left(p_{1}^{*} \varphi \psi w\right)\left(1-p_{2}^{*} t_{1}\right)+(-1)^{n}\left(p_{1}^{*} \gamma w\right)\left(p_{2}^{*} d t_{1}\right)\right),
$$

where

$$
p_{1}: \Delta^{q} \times \Delta^{1} \rightarrow \Delta^{q}, \quad p_{2}: \Delta^{q} \times \Delta^{1} \rightarrow \Delta^{1}
$$

are the projections and $\gamma: \Omega_{q}^{p} \rightarrow \Omega_{q}^{p-1}$ is the mapping of Lemma 5.5. A straightforward computation (using Lemma 5.5) shows that $F$ has the required properties.

If $D: C^{q-1}\left(\Delta\left(R\left[x_{n}\right]\right)\right) \rightarrow C^{q}\left(\Delta\left(R\left[x_{n}\right]\right)\right)$ is the cochain homotopy coming from $F$ above, then

$$
d D+D d=\psi^{*} \varphi^{*}-\mathrm{id}
$$

Hence, by Proposition 8.12 and Lemma 9.2, $C^{*}\left(\Delta\left(R\left[x_{n}\right]\right)\right)$ is splittable.

We complete the inductive step proving Lemma 2.8 by the following

Lemma 9.4. If $i_{*}: H_{*}(A) \rightarrow H_{*}(\mathscr{A} \Delta(A))$ is an isomorphism then

$$
i_{*}: H_{*}(A[y]) \rightarrow H_{*}(\mathscr{A} \Delta(A[y]))
$$

is also an isomorphism.

Proof. According to Theorem 5.6, it is sufficient to show that the composite

$$
j: A[y] \stackrel{i}{\rightarrow} \mathscr{A} \Delta(A[y]) \stackrel{\psi}{\rightarrow} C^{*}(\Delta(A[y]))
$$

induces an isomorphism on homology. By Lemmas 6.12 and 8.3, we may apply the Serre spectral sequence to the fibration

$$
\pi: \Delta(A[y]) \rightarrow \Delta(A)
$$

Define a filtration on $A[y]$ by

$$
F^{p}=\left\{\sum a_{i} y^{i} \in A[y]: \operatorname{dim} a_{i} \geq p\right\} .
$$

We must show that $j$ is filtration preserving.

Suppose $a=\sum a_{i} y^{i}, \operatorname{dim} a_{i} \geq p$, and $w \in \Delta(A[y])_{q}$ with $\pi w \in \Delta(A)_{q}^{p-1}$. We need to show that $j(a)(w)=0$. There is a simplicial mapping $h: \Delta[q] \rightarrow$ $\Delta[p-1]$ and $w^{\prime} \in \Delta(A)_{p-1}$ such that $\pi w=h^{*} w^{\prime}$, where $h^{*}: \Omega_{p-1} \rightarrow \Omega_{q}$. 
Hence,

$$
i(a)(w)=w(a)=w\left(\sum a_{i} y^{i}\right)=\sum w\left(a_{j}\right) w\left(y^{i}\right)=0
$$

since $w\left(a_{i}\right)=h^{*} w^{\prime}\left(a_{i}\right)=0$.

It now follows that $j$ induces a mapping of spectral sequences and

$$
\begin{aligned}
E_{2}^{p, q}(A[y]) & =H_{p}(A) \otimes(R[y])^{q}, \\
E_{2}^{p, q}\left(C^{*}(\Delta(A[y]))\right) & \left.=H^{p}(\Delta(A)) \otimes H^{q}(\Delta(R[y]))\right) .
\end{aligned}
$$

Since the spectral sequences are multiplicative, it suffices to prove that $j$ induces an isomorphism on $E_{2}^{0, q}$ and $E_{2}^{p, 0}$. However, this follows from Lemma 9.3 and the hypothesis on $A$.

\section{Proof of Theorem 2.13}

We begin by proving Theorem 2.13 for $X=K(\pi, n)$. Since $\pi$ is abelian (by definition) and locally euclidean (by assumption), it is the product of groups isomorphic to $Z, Z_{p}=Z / p Z, S^{1}$, or $R$.

Lemma 10.1. For any $n \geq 1$,

$$
\begin{aligned}
H^{*}(K(\pi, n)) & \simeq R[x] & & \text { if } \pi=Z \text { or } R, \\
& =R & & \text { if } \pi=Z_{p} \text { or } S^{1}
\end{aligned}
$$

where $\operatorname{deg} x=n$.

Proof. The case $\pi=R$ is dealt with in Theorem 2.3 and the cases $\pi=Z, Z Z_{p}$ are well known. Suppose $\pi=S^{1}$ and $n=1$. Then, just as in the proof of Lemma 9.1, we see that $H^{*}\left(K\left(S^{1}, 1\right)\right)$ is the classical continuous cohomology of the Lie group $S^{1}$, which is trivial since $S^{1}$ is compact (by the Van Est theorem [24]).

The fact that $H^{*}\left(K\left(S^{1}, n\right)\right)$ is trivial for $n>1$ now follows by induction in the usual way using the continuous cohomology version of the Serre spectral sequence $(\S 8)$ of the TCP

$$
K\left(S^{1}, n\right) \subset C^{n}\left(S^{1}\right) \rightarrow K\left(S^{1}, n+1\right) .
$$

Corollary 10.2. For any $n \geq 1$, we have

$$
\pi^{i}(K(\pi, n))= \begin{cases}0 & \text { if } i \neq n \text { or } \text { if } \pi=Z_{p} \text { or } S^{1} \\ R & \text { if } i=n \text { and } \pi=Z \text { or } R .\end{cases}
$$

This proves Theorem 2.13 for $X=K(\pi, n)$. We next prove the result for $X=X_{n}=E\left(u_{n}\right)$ by induction on $n$.

Suppose $M_{n-1}$ is minimal and $f: X_{n-1} \rightarrow \Delta M_{n-1}$ is a weak equivalence with

$$
\pi^{i}\left(X_{n-1}\right) \simeq \pi^{i}\left(\Delta M_{n-1}\right) \simeq M_{n-1} / \hat{M}_{n-1} .
$$


We can factor $X_{n} \rightarrow X_{n-1}$ into a sequence of TCP with fibre $K(\pi, n)$, where $\pi$ is one of the groups mentioned above. Thus, it is sufficient to consider the case in which $\pi_{n}$ is one of these groups. If $\pi_{n}$ is isomorphic to either $Z_{p}$ or $S^{1}$, then $H^{*}\left(X_{n}\right) \simeq H^{*}\left(X_{n-1}\right)$ and we take $M_{n}=M_{n-1}$.

If $\pi_{n}$ is isomorphic to $Z$ or $R$, we let $\rho: \pi_{n} \rightarrow R$ be the homomorphism taking 1 onto 1 . Let $w \in H^{n+1}\left(\Delta\left(M_{n-1}\right)\right)$ be the image of $u_{n}$ under the composite

$$
H^{n+1}\left(X_{n-1}, \pi_{n}\right) \rightarrow H^{n+1}\left(X_{n-1}, R\right) \simeq H^{n+1}\left(\Delta\left(M_{n-1}\right)\right)
$$

and $v \in H_{n+1}\left(M_{n-1}\right)$ the image of $w$ under the composite

$$
H^{n+1}\left(\Delta\left(M_{n-1}\right)\right) \simeq H_{n+1}\left(\mathscr{A}\left(\Delta\left(M_{n-1}\right)\right)\right) \simeq H_{n+1}\left(M_{n-1}\right) .
$$

Then, if $\tilde{v} \in M_{n-1}$ is a representative for $v \in H_{n+1}\left(M_{n-1}\right)$ we set $M_{n}=$ $M_{n-1}[x]$ with degree $x=n$ and $d x=\tilde{v}$. Comparing Serre spectral sequence shows that $H^{*}\left(X_{n}\right)$ and $H^{*} E(w)$ are isomorphic. In addition, one has $\pi^{i}\left(X_{n}\right) \simeq \pi^{i} E(w)$ and, by Lemma $6.12, E\left(w_{n}\right)$ and $\Delta\left(M_{n}\right)$ are homotopy equivalent. The exact homotopy sequence of the TCP

$$
\Delta(R[x]) \subset \Delta\left(M_{n}\right) \rightarrow \Delta\left(M_{n-1}\right)
$$

yields an isomorphism

$$
\pi^{i}\left(\Delta\left(M_{n}\right)\right) \simeq M_{n} / \hat{M}_{n}
$$

The general case of Theorem 2.3 follows from the observation that for fixed $q$,

$$
H^{q}(X) \simeq H^{q}\left(X_{n}\right) \text { and } \pi^{q}(X) \simeq \pi^{q}\left(X^{n}\right)
$$

for $n$ large enough.

\section{Proof of Theorem 2.23}

We begin with the construction of simplicial sets $X_{1}$ and $X_{2}$ satisfying the conditions of the theorem.

Let $l_{q} \in H^{q}(K(Z, q) ; Z)$ be the generator and let $X_{n, m}$ be the fibration over $K(Z, 2) \vee K(Z, 2)$ induced from the contractible fibration by

$$
f_{n, m}: K(Z, 2) \vee K(Z, 2) \rightarrow K(Z, 4),
$$

where

$$
f_{n, m}^{*}\left(l_{4}\right)=n\left(l_{2} \otimes 1\right)^{2}-m\left(1 \otimes l_{2}\right)^{2} .
$$

Then

$$
H^{*}\left(X_{n, m} ; Q\right)=Q[x, y] /\left\{n x^{2}-m y^{2}, x y\right\},
$$

where $x$ and $y$ have degree 2. If $z=x^{2} / m$, then

$$
H^{2}\left(X_{n, m} ; Q\right) \simeq Q+Q
$$

with basis $x$ and $y$ and

$$
H^{4}\left(X_{n, m} ; Q\right) \simeq Q
$$


with basis $z$. The matrix for quadratic form $H^{2}\left(X_{n, m}\right) \rightarrow H^{4}\left(X_{n, m}\right)$ in this basis is $\left(\begin{array}{cc}m & 0 \\ 0 & n\end{array}\right)$.

Let $M_{n, m}=R[x, y, u, v] \in \mathscr{A} \mathscr{C}$, where $d x=d y=0, d u=n x^{2}-m y^{2}$ and $d v=x y$. In $[3, \S 16]$, it is shown that since, $m x^{2}-m y^{2}, x y$ is an ESP sequence, $M_{n, m}$ is a minimal model for $\mathscr{A}\left(X_{n, m}\right)$.

Let $X_{1}=X_{1,15}$ and $X_{2}=X_{3,5}$. Then the matrices

$$
\left(\begin{array}{cc}
1 & 0 \\
0 & 15
\end{array}\right), \quad\left(\begin{array}{ll}
3 & 0 \\
0 & 5
\end{array}\right)
$$

are not equivalent over $Q$. For if they were equivalent, one would have rational solutions to the equation $3 a^{2}+5 b^{2}=1$, or, equivalently, integer solutions to the equations

$$
3 a^{2}+5 b^{2}=c^{2}
$$

The fact that this is not possible is proved by working mod 3 and using the notion of "infinite descent"; the existence of solutions $a, b, c$ for (11.2) imply the existence of solutions $a^{\prime}, b^{\prime}, c^{\prime}$ with $a^{\prime}<a, b^{\prime}<b, c^{\prime}<c$.

It follows that $H^{*}\left(X_{1} ; Q\right)$ and $H^{*}\left(X_{2} ; Q\right)$ are not isomorphic. However, the matrices (11.1) are equivalent over $R$ (since they have the same signatures). Hence $M_{1,15}$ and $M_{3,5}$ are isomorphic and then $X_{1}$ and $X_{2}$ are equivalent in $\Delta \mathscr{T}_{0 R}$.

We now prove the second assertion of Theorem 2.23. We are indebted to Tsuneo Tamagawa for his help with this proof.

Let $A$ be the free DG algebra over $R$ generated by $x_{1}, \ldots, x_{n} \in A_{2}$, $y_{1}, \ldots, y_{m} \in A_{3}$ and with

$$
\begin{aligned}
& d x_{i}=0, \quad 1 \leq i \leq n, \\
& d y_{i}=\sum_{j \leq k} b_{i}^{j k} x_{j} x_{k}, \quad 1 \leq i \leq m .
\end{aligned}
$$

We say that $A$ has a rational form if there is a DG algebra $A^{\prime}$ over $Q$ and an isomorphism $A \simeq A^{\prime} \otimes_{Q} R$ of DG algebras. Equivalently, $A$ has a rational form if we can choose bases $\tilde{x}_{1}, \ldots, \tilde{x}_{n}$ for $A^{2}, \tilde{y}_{1}, \ldots, \tilde{y}_{m}$ for $A^{3}$ such that

$$
d \tilde{y}_{i}=\sum_{j \leq k} \tilde{b}^{j k} \tilde{x}_{j} \tilde{x}_{k}
$$

with $\tilde{b}_{i}^{j k} \in Q$.

Suppose $A$ has a rational form. Let $P=\left(p_{i j}\right)$ be an invertible $n \times n$ matrix, $Q=\left(q_{i j}\right)$ an invertible $m \times m$ matrix with

$$
x_{i}=\sum p_{i j} \tilde{x}_{j}, \quad \tilde{y}=\sum q_{i j} y_{j} .
$$

Then

$$
d \tilde{y}_{i}=\sum q_{i j} d y_{j}=\sum q_{i j} b_{j}^{k l} p_{k r} p_{l s} \tilde{x}_{r} \tilde{x}_{s}
$$


so

$$
\tilde{b}_{i}^{r s}=\sum_{j, k, l} q_{i j} b_{j}^{k l} p_{k r} p_{l s}
$$

Of course, $b_{i}^{j k}$ can be expressed in terms of $\tilde{b}_{i}^{r s}$ in a similar way.

Let $N=n(n+1) m / 2$. Then there are $N$ of the $b_{i}^{j k}$ and $n^{2}+m^{2}$ of the $p_{i j}, q_{i j}$. We can think of the passage from $\tilde{b}_{i}^{r s}$ to $b_{i}^{j k}$ as defining a mapping $\varphi_{P, Q}: R^{N} \rightarrow R^{N}$ depending on the particular choice of $P$ and $Q$. If we can fix a rational point $\tilde{b} \in Q^{N} \subset R^{N}$, then the set

$$
A^{\prime}(\tilde{b})=\left\{\varphi_{P, Q}(\tilde{b}): P \text { invertible } n \times n, Q \text { invertible } m \times m\right\}
$$

corresponds to all DG algebras $A$ (which of course depend on $b_{i}^{j k}$ ) with $A^{\prime}$ defined by $\tilde{b}$ as their rational form. Thus, the set $\bigcup_{\tilde{b} \in Q} A^{\prime}(\tilde{b})$ corresponds to the set of all $A$ as above which have a rational form.

Now, if $N>n^{2}+m^{2}$ (for example, $n=5, m=2$ ), then $A^{\prime}(\tilde{b})$ is the image of an open set in $R^{n^{2}+m^{2}}$ under a differentiable mapping $R^{n^{2}+m^{2}} \rightarrow R^{N}$. Thus, the set $\bigcup_{\tilde{b} \in Q^{N}} A^{\prime}(\tilde{b})$ cannot be all of $R^{N}$ and any point in its complement corresponds to a real form of $A$ with no rational form.

\section{Proof of Theorem 6.2}

We prove that if $p: E \rightarrow B$ is a fibration in $\Delta \mathscr{T}$, then for any $X \in \Delta \mathscr{T}$, $\mathscr{F}(X, E) \rightarrow \mathscr{F}(X, B)$ is a fibration in $\Delta \mathscr{T}$.

If $I=\left\{i_{1}, \ldots, i_{l}\right\}, 0 \leq i_{1}<\cdots<i_{l} \leq q$, let $\Delta[q, I]$ denote the smallest subsimplicial set of $\Delta[q]$ containing $(0, \ldots, \hat{j}, \ldots, q)$ for $j \in I$. Let $\mathscr{F} E(q, I)$ be the subsimplicial space of $\mathscr{F}(\Delta[q], B) \times \mathscr{F}(\Delta[q, I], E)$ whose $p$ simplices are pairs $(u, v)$, where $u: \Delta[p] \times \Delta[q] \rightarrow B, v: \Delta[p] \times \Delta[q, I] \rightarrow E$, and $u \mid \Delta[p] \times \Delta[q, I]=p v$. The projection induces a map $p_{q, I}: \mathscr{F}(\Delta[q], E) \rightarrow$ $\mathscr{F} E(q, I)$. Note that

$$
E(q, I)=\mathscr{F} E(q, I)_{0}, \quad E_{q}=\mathscr{F}(\Delta[q], E)_{0},
$$

and $p_{q, I}$ in dimension zero coincides with $p_{q_{, I}}$ as previously defined. Furthermore, conditions (iii) and (iv) in the definition of fibration translate into a functional form as follows: If

$$
(u, v) \in E(q-1, I) \subset(\Delta[q-1], B) \times(\Delta[q-1, I], E)
$$

and $j \notin I$, then

$$
\lambda_{q-1, I}(u, v) d_{j}=\lambda_{q, s_{j} I}\left(u d_{j}, v d_{j}\right) .
$$

If $j$ or $j+1 \in I, v: \Delta[q-1] \rightarrow E$ and $u=p v$, then

$$
\lambda_{q, I}\left(u d_{j}, v d_{j} \mid \Delta[q, I]\right)=v d_{j}
$$


Proposition 12.1. The following are equivalent:

(I) $p: E \rightarrow B$ is a fibration in $\Delta \mathscr{T}$.

(II) The maps $p_{q, I}: \mathscr{F}(\Delta[q], E)_{p} \rightarrow \mathscr{F} E(q, I)_{p}$ have continuous sections $\lambda_{I, p}=\lambda_{q, I, p}$ such that if $(u, v) \in \mathscr{F} E(q, I)_{p} \quad(u: \Delta[p] \times \Delta[q] \rightarrow B, v: \Delta[p] \times$ $\Delta[q, I] \rightarrow E)$, then

(i) $\lambda_{I, p-1}\left(u\left(e_{j} \times \mathrm{id}\right), v\left(e_{j} \times \mathrm{id}\right)\right)=\lambda_{I, p}(u, v)\left(e_{j} \times \mathrm{id}\right)$.

(ii) $\lambda_{I, p+1}\left(u\left(d_{j} \times\right.\right.$ id $), v\left(d_{j} \times\right.$ id $\left.)\right)=\lambda_{I, p}(u, v)\left(d_{j} \times\right.$ id $)$.

(iii) If $j$ or $j+1 \in I$, then $\lambda_{I, p}\left(u\left(\mathrm{id} \times d_{j}\right), v\left(\mathrm{id} \times d_{j}\right)\right)=v\left(\mathrm{id} \times d_{j}\right)$ where $v: \Delta[p] \times \Delta[q-1] \rightarrow E$ and $u=p v$.

(iv) If $j \notin I$, then

$$
\lambda_{\bar{s}_{j} I, p}\left(u\left(\mathrm{id} \times d_{j}\right), v\left(\mathrm{id} \times d_{j}\right)\right)=\lambda_{I, p}(u, v)\left(\mathrm{id} \times d_{j}\right) .
$$

(III) There are maps $\lambda_{q, I}: \mathscr{F} E(q, I) \rightarrow \mathscr{F}\left(\Delta_{q}, E\right)$ such that

(i) $p_{q, I} \lambda_{q, I}=$ identity.

(ii) If $j$ or $j+1 \in I$, then

$$
\lambda_{q, I} p_{q, I} \mathscr{F}\left(d_{j}, E\right)=\mathscr{F}\left(d_{j}, E\right)\left(\mathscr{F}\left(d_{j}, E\right): \mathscr{F}(\Delta[q-1], E) \rightarrow \mathscr{F}(\Delta[q], E)\right) .
$$

(iii) If $j \notin I$ and $d_{j}^{*}$ denotes the map induced by $d_{j}: \Delta[q] \rightarrow \Delta[q-1]$, then the following commutes:

$$
\begin{array}{cc}
\mathscr{F}(\Delta[q-1], E) & \stackrel{\lambda_{q-1, I}}{\longrightarrow} \mathscr{F} E(q-1, I) \\
\downarrow_{j}^{*} & \downarrow d_{j}^{*} \\
\mathscr{F}(\Delta[q], E) & \stackrel{\lambda_{q, \bar{s}_{j} l}}{\longrightarrow} \mathscr{F} E\left(q, s_{j} I\right)
\end{array}
$$

(IV) For all $X \in \Delta \mathscr{T}, \mathscr{F}(X, E) \rightarrow \mathscr{F}(X, B)$ is a fibration.

Proof. Statement II implies III since II is simply III reformulated via the definition $\mathscr{F}(X, Y)_{p}=(\Delta[p] \times X, Y)$. The manipulation

$$
\mathscr{F}(X, \mathscr{F}(Y, Z)) \simeq \mathscr{F}(Y, \mathscr{F}(X, Z))
$$

for $X, Y, Z \in \Delta \mathscr{T}$ and $Y$ a finite simplicial set, transforms III into IV. Statement IV implies I by taking $X=\mathrm{pt}$. Thus it remains to verify that I implies II.

Suppose $p: E \rightarrow B$ is a fibration with sections

$$
\lambda_{q, I}: \mathscr{F} E(q, I)_{0} \rightarrow(\Delta[q], E) .
$$

Suppose $(u, v) \in \mathscr{F} E(q, I)_{p}, u: \Delta[p] \times \Delta[q] \rightarrow B, v: \Delta[p] \times \Delta[q, I] \rightarrow E$. We define $\lambda_{I, p}(u, v): \Delta[p] \times \Delta[q] \rightarrow E$ by induction on $q$ and then induction on $p$. For $q=0, \mathscr{F} E(q, I)$ is null since there are no $I$ 's and hence $\lambda_{I, 0}$ is trivially defined. Suppose $\lambda_{I, p}$ has been constructed for $q-1$ satisfying (i)-(iv). For $q$, let $\lambda_{I, 0}=\lambda_{q, I}$ from above. Suppose $\lambda_{I, p-1}$ has been defined. We wish to define $w=\lambda_{I, p}(u, v)$ so that $w$ extends $v, p w=u, w$ varies continuously 
with $(u, v)$ and it satisfies (i)-(iv). Let $\dot{\Delta}[p]$ be the usual simplicial boundary of $\Delta[p]$. Using $\lambda_{I, p-1}$ we extend $v$ to

$$
U_{0}=(\Delta[p] \times \Delta[q, I]) \cup(\dot{\Delta}[p] \times \Delta[q])
$$

so that it satisfies (i). We next choose an order, $\gamma_{1}, \gamma_{2}, \ldots, \gamma_{t}$ of the nondegenerate $p+q$ simplices of $\Delta[p] \times \Delta[q]$ and extend $w$ over each $\gamma_{i}$ by induction on $i$ using the sections $\lambda_{p+q, I}$ for $E \rightarrow B$. The main technical difficulties involved in this proof consist in choosing this order so that (i)-(iv) hold. We next define this order and prove three lemmas about it.

Note that if $(\tau, \sigma) \in(\Delta[p] \times \Delta[q])_{p+q}$ is nondegenerate, $\sigma$ determines $\tau$. If $\sigma=\left(j_{0}, j_{1}, \ldots, j_{p+q}\right)$ and $\tau=\left(k_{0}, \ldots, k_{p+q}\right)$, then $k_{0}=0, k_{i+1}=k_{i}+1$ if $j_{k+1}=j_{i+1}$ and $k_{i+1}=k_{i}$ if $j_{i+1}=j_{i}+1$. Let $\hat{\sigma}$ denote this $\tau$, determined by $\sigma$ and let $V_{q}^{p}$ denote all $p+q$ simplices $\sigma$ of $\Delta[q]$ arising in this way, that is, all $p+q$ simplices involving all of $\{0,1, \ldots, q\}$. We define an order on $\{0,1, \ldots, q\}$ with respect to $I=\left(i_{1}, i_{2}, \ldots, i_{l}\right)$ by writing it in the order $\left(j_{0}, j_{1}, \ldots, j_{q-l}, i_{1}, i_{2}, \ldots, i_{l}\right), 0 \leq j_{0}<j_{1}<\cdots<j_{q-l} \leq q, j_{t} \notin I$. Order the simplices in $V_{q}^{p}$ lexiographically with respect to the above $I$ order on $\{0,1, \ldots, q\}$ and order the simplices $(\hat{\sigma}, \sigma), \sigma \in V_{q}^{p}$, according to the $I$ order of $\sigma$. We denote these orderings by " $<_{I}$ ".

Let $l(i, \sigma)$ be the integer $l$, where $i_{l}$ is the last occurrence of $i$ in $\sigma$. The following is trivial to check.

Lemma 12.2. If $\sigma \in V_{q}^{p}$, then

$$
\left(d_{i} \times \mathrm{id}\right)(\hat{\sigma}, \sigma)=s_{l}\left(\partial_{l} \hat{\sigma}, \partial_{l} \sigma\right),
$$

where $l=l(\hat{\sigma}, i)$, and

$$
\left(\mathrm{id} \times d_{i}\right)(\hat{\sigma}, \sigma)=s_{l}\left(\partial_{l} d_{i} \hat{\sigma}, \partial_{l} d_{i} \sigma\right),
$$

where $l=l(\sigma, i)$.

If $\sigma \in V_{q}^{p}$, let $T_{l} \sigma \in V_{q}^{p}$ be defined as follows: If $i_{l}=i,\left(i_{l-1}, i_{l}, i_{l+1}\right)$ is one of $(i, i, i),(i-1, i, i),(i, i, i+1)$ or $(i-1, i, i+1)$. Let $T_{l} \sigma$ be formed from $\sigma$ by changing $i_{l}$ in the cases above, to $i, i-1, i+1, i$, respectively. Let

$$
I(\sigma, I)=\left\{l \mid T_{l}(\sigma)<_{I} \sigma\right\} \cup\left\{l \mid \partial_{l} \sigma \in \Delta[q, I] \text { or } \partial_{l} \hat{\sigma} \in \dot{\Delta}[p]\right\} .
$$

The second factor in this union is

$$
\left\{l \mid T_{l} \sigma=\sigma \text { and if } i_{l} \notin I, i_{l+1}=i_{l}\right\} .
$$

The proof of the following is a bit tedious but straightforward.

Lemma 12.3. The set $I(\sigma, I)$ is nonnull and $\neq(0,1, \ldots, p+q)$. If $i$ or $i+1 \in I$, then $l(\sigma, i)$ or $l(\sigma, i)+1 \in I(\sigma, I)$. If $i$ and $i+1 \notin I$, then $s_{l} I\left(\partial_{l} d_{i} \sigma, I\right)=I\left(\sigma, s_{i} I\right)$, where $l=l(\sigma, i)$. If $l=l(\hat{\sigma}, i)$ and $l$ and $l+1 \notin$ $I(\sigma, I)$, then $s_{l} I\left(\partial_{l} \sigma, I\right)=I(\sigma, I)$. 
For a simplex $\alpha$, let $\bar{\alpha}$ denote the simplicial set of all faces and degeneracies of $\alpha$. For $\sigma \in V_{q}^{p}$, let

$$
U_{\sigma}^{I}=\Delta[p] \times \Delta[q, I] \cup(\Delta[\dot{p}] \times \Delta[q]) \cup \bigcup_{\alpha<{ }_{1} \sigma} \overline{(\hat{\alpha}, \alpha)},
$$

where the $\alpha$ 's are in $\Omega_{q}^{p}$. We prove

Lemma 12.4. $U_{\sigma}^{I} \cap \overline{(\hat{\sigma}, \sigma)}=\bigcup_{i \in I(\sigma, I)} \partial_{i} \overline{(\hat{\sigma}, \sigma)}$.

Proof. Recall $T_{l} \sigma$ differs from $\sigma$ only in the $l$ th place and hence $\partial_{l}\left(T_{l} \sigma\right)=$ $\partial_{l} \sigma$. Thus if $T_{l}(\sigma)<_{I} \sigma$, then $\partial_{l}(\hat{\sigma}, \sigma) \in U_{\sigma}^{I}$. The definition of $I(\sigma, I)$ thus shows that the right side of Lemma 12.4 is contained in the left side. Suppose $(\hat{\tau}, \tau)$ is a nondegenerate simplex in the left side of 12.4 and not the face of such a simplex. We show that $(\hat{\tau}, \tau)=\partial_{i}(\hat{\sigma}, \sigma)$ for some $i \in I(\sigma, I)$. Suppose $\tau \in \Delta[q, I]$. Let the last occurrence of $i_{1}\left(I=\left(i_{1}, i_{2}, \ldots\right)\right)$ in $\sigma$ occur at $l$. Then $\tau$ is a face of $\partial_{l} \sigma$ and $l \in I(\sigma, I)$ and hence $\tau=\partial_{l} \sigma$. Suppose $\tau$ is a face of $(\hat{\alpha}, \alpha) \in\left(e_{j}(\Delta[p-1])\right) \times \Delta[q]$. Let $l$ be the last place at which $j$ occurs in $\hat{\alpha}$. Then $l \in I(\sigma, I)$ or $l-1 \in I(\sigma, I)$ and the $l-1$ entry of $\hat{\alpha}$ is also $j$. In either case $\tau$ is a face of $\partial_{i} \sigma$ where $i \in I(\sigma, I)$. Suppose $\tau$ is a face of $\sigma^{\prime}, \sigma^{\prime}<_{I} \sigma$ and suppose $\tau \neq \partial_{i} \sigma$, all $i \in I(\sigma, I)$. We show this leads to a contradiction which will complete the proof. Since $\tau$ is a face of $\sigma, \tau=\partial_{j_{1}} \partial_{j_{2}} \cdots \partial_{j_{t}} \sigma, j_{1}<j_{2}<\cdots<j_{t}$ and $j_{s} \notin I(\sigma, I)$, all $s$. Suppose $j$ is the $j_{s}$ entry of $\hat{\sigma}$ and $b$ is the $j_{s}$ entry of $\sigma$, i.e. $(b, j)$ is the $j_{s}$ vertex of $(\hat{\sigma}, \sigma)$. Since $j_{s} \notin I(\sigma, I)$, the possible $j_{s}-1, j_{s}, j_{s}+1$ vertices of $(\hat{\sigma}, \sigma)$ are $(b, j-1),(b-j),(b, j+1) a, j \notin$ $I$ which we call type $A$, or $(b-1, j),(b, j),(b, j+1), j \notin I$ or $j$ and $j+1 \in I$; or $(b, j-1),(b, j),(b+1, j), j-1 \in I$ and $j \notin I$, which we call type $B$. Suppose $j_{s}$ is the first occurrence of a type A situation; $\partial_{j_{s}} \sigma$ omits all occurrences of $j$ in $\sigma$ and $(b, j-1)$ is not omitted by $\partial_{j_{s-1}}$ because it cannot be of type B. Hence $(b, j-1)$ must be in $\left(\hat{\sigma}^{\prime}, \sigma^{\prime}\right)$ and $j \in \sigma$ and thus $(b, j) \in\left(\hat{\sigma}^{\prime}, \sigma^{\prime}\right)$ which contradicts the maximality of $\tau$. Hence there are no type A situations. Being all of type B implies that for all $s, j_{s+1}>j_{s}+1$. But to form $\sigma^{\prime}$ from $\tau$, each vertex omitted from $\sigma$ must be replaced by a vertex and the only possible replacements are bigger in the $I$ order. Hence $\sigma^{\prime}>\sigma$, a contradiction.

We now return to defining $w: \Delta[p] \times[q] \rightarrow E$. We define $w \mid U_{\sigma}, \sigma \in V_{q}^{p}$ by induction on the $I$ order of $V_{q}^{p}$. Suppose $w \mid U$ has been defined. Let $h_{\sigma}: \Delta[p+q] \rightarrow \Delta[p] \times \Delta[p]$ be the unique map such that $h_{\sigma}(0, \ldots, p+q)=$ $(\hat{\sigma}, \sigma)$. Let $w \mid U_{\sigma} \cup \bar{\sigma}$ be the unique map extending a $w \mid U_{\sigma}$ and satisfying

$$
w h_{\sigma}=\lambda_{p+q, I(\sigma, I)}\left(u h_{\sigma},\left(w \mid U_{\sigma}\right)\left(h_{\sigma} \mid \Delta[p+q, I(\sigma, I)]\right)\right) .
$$

Continuing by induction on $\sigma$ we obtain $w: \Delta[p] \times \Delta[q] \rightarrow E$, which by construction satisfies $(i)$ and varies continuously with $(u, v)$. Note that $\lambda_{I, p}(u, v)$ is continuous because its range and domain are subspaces of products of $B_{q}$ 's 
and $E_{q}$ 's and the maps are complicated combinations of the face and degeneracy operations and the maps $\lambda_{q, I}$ from $E \rightarrow B$.

We verify that $w$ satisfies (ii)-(iv) on $U_{\sigma}$ by induction on $\sigma$.

Proof of (ii). Consider

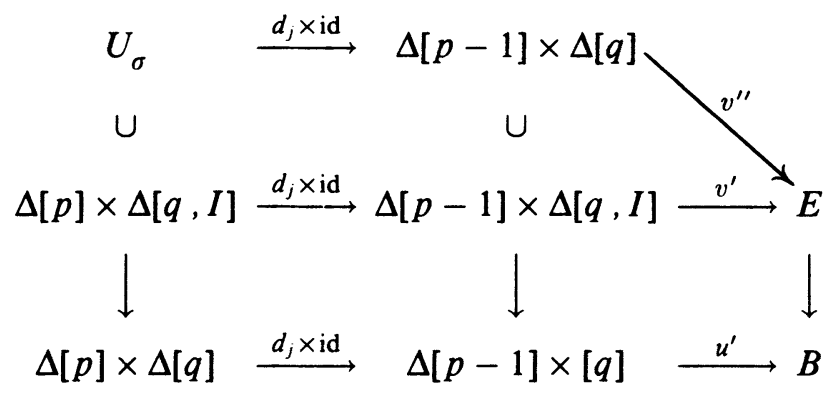

where $v^{\prime \prime}=\lambda_{I, p-1}\left(u^{\prime}, v^{\prime}\right)$. We wish to show that if $w$ is constructed from $u=$ $u^{\prime}\left(d_{j} \times \mathrm{id}\right)$ and $v=v^{\prime}\left(d_{j} \times \mathrm{id}\right)$, then $w=v^{\prime \prime}\left(d_{j} \times \mathrm{id}\right)$. We show that

$$
w\left|u_{\sigma}^{\prime}=v^{\prime \prime}\left(d_{j} \times \mathrm{id}\right)\right| U_{\sigma}
$$

by induction on $\sigma$. The first step in this induction is true because $\lambda_{I, p-1}$ satisfies (ii). Suppose the above is true for $U_{\sigma}$ and $\sigma^{\prime}$ is the successor of $\sigma$. Then $U_{\sigma^{\prime}}=U_{\sigma} \cup \hat{\sigma}$ and hence we must show that $w h_{\sigma}=v^{\prime \prime}\left(I_{j} \times \mathrm{id}\right) h_{\sigma}$. By Lemma 12.2 , if $l=l(\hat{\sigma}, j)$,

$$
\left(d_{j} \times \mathrm{id}\right) h_{\sigma}=h_{\partial_{l} \sigma} d_{l}
$$

Hence

$$
\begin{aligned}
w h_{\sigma} & =\lambda_{p+q, I(\sigma, I)}\left(u h_{\sigma},\left(w \mid U_{\sigma}\right)\left(h_{\sigma} \mid \Delta[p+q, I(\sigma, I)]\right)\right) \\
& =\lambda_{p+q, I(\sigma, I)}\left(u^{\prime} h_{\partial_{l} \sigma} d_{l}, v^{\prime \prime} h_{\partial_{l} \sigma} d_{l} \mid \Delta[p+q, I(\partial, I)]\right) .
\end{aligned}
$$

Suppose $l$ or $l+1 \in I(\sigma, I)$. Then

$$
w h_{\sigma}=v^{\prime \prime} h_{\partial_{l} \sigma} d_{l}=v^{\prime \prime}\left(d_{j} \times \mathrm{id}\right) h_{\sigma} .
$$

Suppose $l$ and $l+1 \notin I(\partial, I)$. Then

$$
\begin{aligned}
w h_{\sigma} & =\lambda_{s_{l} I\left(\partial_{l} \sigma, I\right)}\left(u^{\prime} h_{\partial_{l} \sigma} d_{l}, v^{\prime \prime} h_{\partial_{l} \sigma} d_{l}\right) \\
& =\lambda_{I\left(\partial_{l} \sigma, I\right)}\left(u^{\prime} h_{\partial_{l} \sigma}, v^{\prime \prime} h_{\partial_{l} \sigma}\right) d_{l} \\
& =\lambda_{I, p-1}\left(u^{\prime}, v^{\prime}\right) h_{\partial_{l} \sigma} d_{l} \\
& =v^{\prime \prime}\left(d_{j} \times \mathrm{id}\right) h_{\sigma} .
\end{aligned}
$$


The proof of (iii) follows the same line of argument as used for (ii) starting from the diagram

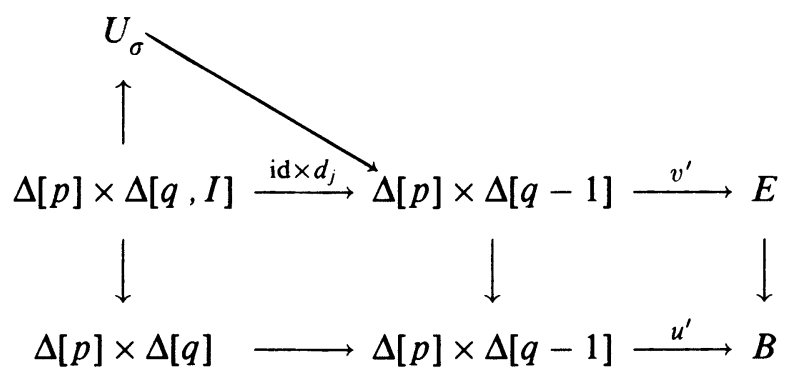

where $j$ or $j+1 \in I$. Let $u=u^{\prime}\left(\mathrm{id} \times d_{j}\right)$ and $v=v^{\prime}\left(\mathrm{id} \times d_{j}\right)$. We need to show that if $w=\lambda_{I, p}(u, v)$, then

$$
w h_{\sigma}=v\left(\mathrm{id} \times d_{j}\right) h_{\sigma} .
$$

(Again we use induction on $\sigma$.) Let $l=l(\sigma, i)$. Then by Lemma $12.3 l$ or $l+1 \in I(\sigma, I)$ and by Lemma 12.2

$$
\left(\mathrm{id} \times d_{j}\right) h_{\sigma}=h_{\sigma^{\prime}} d_{l} \quad \text { where } \sigma^{\prime}=\partial_{l}\left(d_{i} \sigma\right) .
$$

Hence

$$
\begin{aligned}
w h_{\sigma} & =\lambda_{I(\sigma, I)}\left(u^{\prime} h_{\sigma^{\prime}} d_{l}, v^{\prime} h_{\sigma^{\prime}} d_{l}\right) \\
& =v^{\prime} h_{\sigma^{\prime}} d_{l}=v^{\prime}\left(\mathrm{id} \times d_{j}\right) h_{\sigma^{\prime}} .
\end{aligned}
$$

Proof of(iv). Here the diagram is as follows ( $j$ and $\left.j+1 \notin s_{j} I\right)$ :



Again, using Lemmas 12.2 and 12.3, one has

$$
\begin{aligned}
w h_{\sigma} & =\lambda_{s_{l} I\left(\sigma^{\prime}, I\right)}\left(u^{\prime} h_{\sigma^{\prime}} d_{l}, v^{\prime} h_{\sigma^{\prime}} d_{l}\right) \\
& =\lambda_{I\left(\sigma^{\prime}, I\right)}\left(u^{\prime} h_{\sigma^{\prime}}, v^{\prime} h_{\sigma^{\prime}}\right) d_{l} \\
& =v^{\prime \prime} h_{\sigma^{\prime}} d_{l}=v^{\prime \prime}\left(\mathrm{id} \times d_{j}\right) h_{\sigma} .
\end{aligned}
$$

This completes the proof of Proposition 12.1.

\section{REFERENCES}

1. R. G. Bartle and L. M. Graves, Mappings between function spaces, Trans. Amer. Math. Soc. 72 (1952), 400-413.

2. A. Borel and N. Wallach, Continuous cohomology, discrete subgroups, and representations of reductive groups, Princeton Univ. Press, Princeton, N.J., 1980. 
3. A. K. Bousfield and V. K. A. M. Gugenheim, On PL de Rham theory and rational homotopy type, Mem. Amer. Math. Soc. No. 179 (1976).

4. A. K. Bousfield and D. M. Kan, Homotopy limits, completions and localizations, Lecture Notes in Math., vol. 304, Springer-Verlag, Berlin, 1972.

5. E. H. Brown and R. H. Szczarba, Continuous cohomology, Contemp. Math., vol. 58, Amer. Math. Soc., Providence, R.I., 1987, pp. 15-19.

6. P. Deligne, P. Griffiths, J. Morgan, and D. Sullivan, Real homotopy theory of Kähler manifolds, Invent. Math. 29 (1975), 245-274.

7. A. Dold and D. Puppe, Homologie nicht-additiven Functoren, Anwendungen, Ann. Inst. Fourier (Grenoble) 11 (1961), 202-312.

8. A. Haefliger, Feuilletages sur les variétés ouvertes, Topology 9 (1970), 183-194.

9. __ Homotopy and integrability, Manifolds (Amsterdam, 1970), Lecture Notes in Math., vol. 197, Springer-Verlag, 1970, pp. 133-163.

10. __ Cohomology of Lie algebras and foliations, Sympos. Differential and Algebraic Topology, Rio de Janeiro, Lecture Notes in Math., vol. 652, Springer-Verlag, 1976, pp. 1-12.

11. _ Differentiable cohomology, Cours donné au CIME, 1976.

12. P. J. Hilton and S. Wylie, Homology theory, Cambridge Univ. Press, Cambridge, 1962.

13. J. P. May, Simplicial objects in algebraic topology, Univ. of Chicago Press, Chicago, Ill., 1982.

14. E. Michael, Selected-selection theorems, Amer. Math. Monthly 63 (1956), 233-238.

15. J. Milnor, Construction of universal bundles. II, Ann. of Math. (2) 63 (1956), 430-436.

16. M. A. Mostow, Continuous cohomology of spaces with two topologies, Mem. Amer. Math. Soc. No. 174 (1976).

17. D. G. Quillen, Rational homotopy theory, Ann. of Math. (2) 90 (1969), 205-295.

18. G. Segal, Classifying spaces and spectral sequences, Inst. Hautes Études Sci. Publ. Math. 34 (1968), 105-112.

19. __, Categories and cohomology theories, Topology 13 (1974), 293-312.

20. N. E. Steenrod, Products of cocycles and extensions of mappings, Ann. of Math. (2) 48 (1947), 290-320.

21. __, A convenient category of topological spaces, Michigan Math. J. 14 (1967), 133-152.

22. W. Thurston, The theory of foliations in codimension greater than one, Comment. Math. Helv. 49 (1974), 214-231.

23. F. Treves, Topological vector spaces, distributions, and kernels, Academic Press, New York, 1967.

24. W. T. Van Est, Group cohomology and Lie algebra cohomology in Lie groups. I, II, Nederl. Akad. Wetensch. Proc. Ser. A 56 (1953), 484-492; 493-504.

25. J. L. Dupont, Simplicial de Rham cohomology and characteristic classes of flat bundles, Topology 15 (1976), 233-245.

26. D. Sullivan, Infinitesimal computations in topology, Inst. Hautes Études Sci. Publ. Math. 47 (1977), 269-331.

Department of Mathematics, Brandeis University, Waltham, Massachusetts 02254

Department of Mathematics, Yale University, Box 2155 Yale Station, New Haven, CONNECTICUT 06520 